Linear wave propagation for resistive relativistic magnetohydrodynamics

A. Mignone, G. Mattia, and G. Bodo

Citation: Physics of Plasmas 25, 092114 (2018); doi: 10.1063/1.5048496

View online: https://doi.org/10.1063/1.5048496

View Table of Contents: http://aip.scitation.org/toc/php/25/9

Published by the American Institute of Physics 


\title{
Linear wave propagation for resistive relativistic magnetohydrodynamics
}

\author{
A. Mignone, ${ }^{1}$ G. Mattia, ${ }^{1}$ and G. Bodo ${ }^{2}$ \\ ${ }^{1}$ Dipartimento di Fisica, University di Torino, via Pietro Giuria 1, I-10125 Torino, Italy \\ ${ }^{2}$ INAF, Osservatorio Astrofisico di Torino, Strada Osservatorio 20, 10025 Pino Torinese, Italy
}

(Received 15 July 2018; accepted 4 September 2018; published online 28 September 2018)

\begin{abstract}
We present a linear mode analysis of the relativistic magnetohydrodynamics equations in the presence of finite electrical conductivity. Starting from the fully relativistic covariant formulation, we derive the dispersion relation in the limit of small linear perturbations. It is found that the system supports ten wave modes which can be easily identified in the limits of small or large conductivities. In the resistive limit, matter and electromagnetic fields decouple and solution modes approach pairs of light and acoustic waves as well as a number of purely damped (non-propagating) modes. In the opposite (ideal) limit, the frozen-in condition applies and the modes of propagation coincide with a pair of fast magnetosonic, a pair of slow and Alfvén modes, as expected. In addition, the contact mode is always present and it is unaffected by the conductivity. For finite values of the conductivity, the dispersion relation gives rise to either pairs of opposite complex conjugate roots or purely imaginary (damped) modes. In all cases, the system is dissipative and also dispersive as the phase velocity depends nonlinearly on the wavenumber. Occasionally, the group velocity may exceed the speed of light although this does not lead to superluminal signal propagation. Published by AIP Publishing.

https://doi.org/10.1063/1.5048496
\end{abstract}

\section{INTRODUCTION}

The dynamics of relativistic plasmas are of great interest both in the laboratory, as in the case of laser produced plasmas, and for high energy astrophysics. The large scale properties of such plasmas can be described by using the magnetohydrodynamics (MHD) approximation, whose relativistic extension has been developed by Lichnerowicz ${ }^{1}$ and Anile ${ }^{2}$ paralleling the well-studied non-relativistic version. Relativistic MHD (RMHD henceforth) has been employed over the last few decades to describe the dynamics of such systems well in their nonlinear regimes, particularly through the use of numerical simulations and remarkable progress has been made in the development of numerical methods for the RMHD equations (see, e.g., Refs. 3-8). Even though the ideal limit, where dissipative effects are neglected, captures effectively the dynamics in most of the situations, there are cases in which resistivity plays a fundamental role, magnetic reconnection is a notable example. Therefore, in the last few years strong interest has been devoted to the resistive RMHD equations and to finding robust and accurate numerical schemes for their solution. ${ }^{9,10}$

The ideal RMHD linear wave dynamics are well known: just as in the case of classical MHD, the plasma supports slow, fast magnetosonic and Alfvén waves and expressions for the wave speeds have been obtained and used, for example, in numerical schemes for relativistic magnetofluid codes, see Refs. 3-6,11,12 and references therein. A compendium of the properties of such linear waves can be found, for example, in Keppens and Meliani. ${ }^{13}$ The properties of linear waves in the resistive case are less well known and the purpose of this paper is to give a comprehensive analysis of such waves. The results presented in this paper, in addition to being of interest per se, can be particularly relevant for the construction of numerical schemes for the resistive RMHD equations.
The propagation of electromagnetic waves in resistive pair plasmas has been presented by Koide ${ }^{14}$ using a one-fluid theory derived from the relativistic two-fluid equations. An approximate dispersion relation for the resistive RMHD equations, that considers only transverse wave propagation (i.e., Alfvén waves) in the magnetic field direction, has been derived in Appendix of Ref. 15 in the development of a numerical scheme. More recently, a linear analysis of the resistive RMHD equations has been presented by Del Zanna et al. ${ }^{16}$ in the context of tearing mode instability by investigating the stability of an initial force-free current field. In their study, the authors assume an incompressible plasma and neglect Ampere's law by assuming an electric field which includes the usual convective and diffusive contributions. In the present work, instead, we present an extensive normal mode analysis of the resistive RMHD equations by retaining the complete form of the equations. In the presence of resistivity, the RMHD equations take the form of hyperbolic equations with relaxation terms, ${ }^{10,17-19}$ this leads to several modifications of the wave properties. In addition to introducing wave damping (as one would expect), resistivity leads to other qualitative changes in the wave properties as well. As in all hyperbolic systems with relaxation, we can distinguish two regimes: ${ }^{18}$ at small wavenumbers resistivity tends to be negligible and the system supports standard RMHD waves, i.e., slow, fast magnetosonic and Alfvén; at large wavenumbers, instead, Maxwell equations decouple from the fluid equations and the system supports light and sound waves. For intermediate wavenumbers, connecting these two regimes, the system becomes dispersive.

The plan of the paper is the following. In Sec. II, starting from the full covariant form of the resistive relativistic MHD (RRMHD) equations, we carry out the normal mode analysis in the limit of small perturbations and obtain the characteristic 
polynomial whose roots give the desired dispersion relation. In Sec. III, we provide asymptotic solutions to the dispersion relations in the resistive and ideal limits. In Sec. IV, the solutions of the dispersion relation are analyzed for finite values of the conductivity and for different values of the parameters. Conclusions are finally drawn in Sec. V.

\section{EQUATIONS}

\section{A. The resistive relativistic MHD equations}

Our starting point is the covariant equations of resistive relativistic MHD which follow from the conservation of particle number density and stress-energy tensor coupled to the Maxwell's equations of classical electromagnetism, see Refs. 1,9,10 and references therein. Using a system of units, where $c=4 \pi=1$, we have

$$
\left\{\begin{array}{l}
\partial_{\alpha}\left(n u^{\alpha}\right)=0 \\
\partial_{\beta}\left(\mathrm{T}^{\alpha \beta}\right)=0 \\
\partial_{\beta} \mathrm{F}^{\alpha \beta}=-J^{\alpha} \\
\partial_{\beta}{ }^{*} \mathrm{~F}^{\alpha \beta}=0
\end{array},\right.
$$

where $\mathrm{F}^{\alpha \beta}$ is the electromagnetic tensor $\left(\mathrm{F}^{0 i}=-\mathrm{F}^{i 0}=-E_{i}\right.$, $\left.\mathrm{F}^{i j}=-\epsilon^{i j k} B_{k}\right),{ }^{*} \mathrm{~F}^{\alpha \beta}$ is its dual, and $J^{\alpha}$ is the four-current vector.

The stress-energy tensor for the composite system fluidelectromagnetic fields can be written as $T^{\alpha \beta}=T_{\text {fluid }}^{\alpha \beta}+T_{\mathrm{em}}^{\alpha \beta}$, where

$$
\left\{\begin{array}{l}
\mathrm{T}_{\text {fluid }}^{\alpha \beta}=w u^{\alpha} u^{\beta}-p \mathrm{~g}^{\alpha \beta} \\
\mathrm{T}_{\mathrm{em}}^{\alpha \beta}=\mathrm{g}^{\alpha \mu} \mathrm{F}_{\mu \lambda} \mathrm{F}^{\lambda \beta}+\frac{1}{4} \mathrm{~F}_{\mu \nu} \mathrm{F}^{\mu \nu} \mathrm{g}^{\alpha \beta}
\end{array}\right.
$$

are the fluid and electromagnetic tensors, respectively, $w$ is the gas enthalpy, $u^{\alpha}=\gamma(1, \boldsymbol{v})$ is the fluid four-velocity, $p$ is the gas pressure, and $g^{\alpha \beta}$ is the metric tensor.

The explicit form of the four-current vector is defined by Ohm's law and accounts only for the plasma resistivity $\eta$ $=1 / \sigma$, where $\sigma$ is the electrical conductivity ${ }^{1,9}$

$$
J^{\alpha}=\frac{1}{\eta} \mathrm{F}^{\alpha \mu} u_{\mu}+q_{0} u^{\alpha},
$$

where $q_{0}=-J^{\alpha} u_{\alpha}$ is the electric charge density in the fluid rest frame. Note that the fluid charge $q$ and current density $\boldsymbol{J}$ in the lab frame are given by the temporal and spatial components of the four-current, respectively,

$$
\begin{gathered}
q \equiv J^{0}=\sigma(\boldsymbol{E} \cdot \boldsymbol{u})+q_{0} \gamma \\
\boldsymbol{J} \equiv J^{i}=\gamma \sigma[\boldsymbol{E}+\boldsymbol{v} \times \boldsymbol{B}-(\boldsymbol{E} \cdot \boldsymbol{v}) \boldsymbol{v}]+q \boldsymbol{v} .
\end{gathered}
$$

Projecting Eq. (1) in the directions parallel and perpendicular to any time-like vector $n^{\mu}$, we obtain the three-dimensional form of the resistive relativistic magnetohydrodynamics (RRMHD henceforth) which, after simple manipulations, can be written as

$$
\frac{\partial(\rho \gamma)}{\partial t}+\nabla \cdot(\rho \gamma \boldsymbol{v})=0
$$

$$
\begin{gathered}
\frac{\partial}{\partial t}\left(w \gamma^{2} \boldsymbol{v}\right)+\nabla \cdot\left(w \gamma^{2} \boldsymbol{v} \boldsymbol{v}\right)+\nabla p=q \boldsymbol{E}+\boldsymbol{J} \times \boldsymbol{B} \\
\frac{\partial \boldsymbol{B}}{\partial t}+\nabla \times \boldsymbol{E}=0 \\
\frac{\partial \boldsymbol{E}}{\partial t}-\nabla \times \boldsymbol{B}=-\boldsymbol{J} \\
\frac{\partial}{\partial t}\left(w \gamma^{2}-p\right)+\nabla \cdot\left(w \gamma^{2} \boldsymbol{v}\right)=\boldsymbol{J} \cdot \boldsymbol{E}
\end{gathered}
$$

where $\rho=n m$ is the rest-mass density, $\gamma=\left(1-\boldsymbol{v}^{2}\right)^{-\frac{1}{2}}$ is the fluid Lorentz factor, $\boldsymbol{v}$ is the fluid velocity, $\boldsymbol{E}$ and $\boldsymbol{B}$ are the electric and magnetic field vectors, and $w$ and $p$ are the gas enthalpy and pressure, respectively.

The temporal components of the third and fourth equations in (1) yield the time-independent Maxwell's relations for the field divergences

$$
\nabla \cdot \boldsymbol{E}=q, \quad \nabla \cdot \boldsymbol{B}=0 .
$$

Finally, an equation of state $(\mathrm{EoS})$, in the form $w=w(\rho, p)$, must be provided for appropriate closure.

\section{B. Normal mode analysis}

The equilibrium state consists of a homogeneous plasma at rest with constant density and pressure $\rho_{0}$ and $p_{0}$, respectively. The system is threaded by a constant and uniform magnetic $\boldsymbol{B}_{0}$, while the electric field must vanish in this frame: $\boldsymbol{E}_{0}=\mathbf{0}$.

Equations (6)-(10) are linearized assuming plane wave perturbations in the form $V_{1} \propto \epsilon e^{i(\boldsymbol{k} \cdot \boldsymbol{x}-\omega t)}$, where $V$ is any of the fluid variables, $\epsilon$ is a small amplitude, $\omega$ is the (complex) frequency, and $\boldsymbol{k}$ is the wavevector. By retaining only terms of order one, we have

$$
\left\{\begin{array}{l}
-i \omega \rho_{1}+i \rho_{0} \boldsymbol{k} \cdot \boldsymbol{v}_{1}=0 \\
-i \omega w_{0} \boldsymbol{v}_{1}+i \boldsymbol{k} p_{1}=\boldsymbol{J}_{1} \times \boldsymbol{B}_{0} \\
-i \omega \boldsymbol{B}_{1}+i \boldsymbol{k} \times \boldsymbol{E}_{1}=0 \\
-i \omega \boldsymbol{E}_{1}-i \boldsymbol{k} \times \boldsymbol{B}_{1}=-\boldsymbol{J}_{1} \\
-i \omega\left[\left(w_{p}^{\prime}-1\right) p_{1}+w_{\rho}^{\prime} \rho_{1}\right]+w_{0} i \boldsymbol{k} \cdot \boldsymbol{v}_{1}=0 .
\end{array}\right.
$$

Here, $\boldsymbol{J}_{1}=\sigma\left[\boldsymbol{E}_{1}+\boldsymbol{v}_{1} \times \boldsymbol{B}_{0}\right]$ is the perturbation of the current density. From the third equation, we always have $\boldsymbol{B}_{1} \cdot \boldsymbol{E}_{1}=0$ that is, magnetic and electric field perturbations are always orthogonal. In addition, the divergence-free condition for magnetic field requires $\boldsymbol{k} \cdot \boldsymbol{B}_{1}=0$. Also, the Lorentz factor is a second-order quantity $\left(\gamma \approx O\left(\epsilon^{2}\right)\right)$ and the charge density $q \approx i \boldsymbol{k} \cdot \boldsymbol{E}_{1}$ appears only through second (or higher) order terms in $\epsilon$. Both quantities, therefore, can be neglected.

Without the loss of generality, the equilibrium magnetic field is taken to lie in the $x-y$ plane: $\boldsymbol{B}_{0}=\left(B_{0 x}, B_{0 y}, 0\right)$ and we the wavevector $\boldsymbol{k}$ along the $x$ direction, $\boldsymbol{k} \equiv k \hat{\mathrm{e}}_{x}$. The linearized RRMHD equations (12) can then be written as a homogenous $10 \times 10$ linear system 


$$
\mathrm{A}\left(\begin{array}{l}
\rho_{1} \\
\boldsymbol{v}_{1} \\
\boldsymbol{B}_{t 1} \\
\boldsymbol{E}_{1} \\
p_{1}
\end{array}\right)=0
$$

where the matrix A is given, in compact form, by

$$
\mathbf{A}=\left[\begin{array}{cccccc}
-\lambda & \rho_{0} \boldsymbol{e}_{x}^{\top} & 0 & 0 & \mathbf{0}^{\top} & 0 \\
\mathbf{0} & \mathrm{T} & \mathbf{0} & \mathbf{0} & \mathrm{M} & \boldsymbol{e}_{x}^{\top} \\
0 & \mathbf{0}^{\top} & -\lambda & 0 & -\hat{\boldsymbol{e}}_{z}^{\top} & 0 \\
0 & \mathbf{0}^{\top} & 0 & -\lambda & \hat{\boldsymbol{e}}_{y}^{\top} & 0 \\
\mathbf{0} & -\mathrm{M} & -\hat{\boldsymbol{e}}_{z} & \hat{\boldsymbol{e}}_{y} & \mathrm{D} & \mathbf{0} \\
-\lambda w_{0, \rho}^{\prime} & w_{0} \boldsymbol{e}_{x}^{\top} & 0 & 0 & 0^{\top} & -\lambda\left(w_{0, p}^{\prime}-1\right)
\end{array}\right] .
$$

In the previous expression, $\lambda=\omega / k \in \mathbb{C}$ is the (complex) eigenvalue, while $\mathrm{T}, \mathrm{M}$, and $\mathrm{D}$ are $3 \times 3$ matrices with components

$$
\mathrm{T}_{i j}=-\left(w_{0} \lambda+i \tilde{\sigma} B_{0}^{2}\right) \delta_{i j}+i \tilde{\sigma} B_{0 i} B_{0 j}
$$

and

$$
\mathrm{M}_{i j}=i \tilde{\sigma} \varepsilon_{i j k} B_{0 k}, \quad \mathrm{D}_{i j}=\operatorname{diag}(-\lambda+i \tilde{\sigma}),
$$

where $\epsilon_{i j k}$ is the Levi-Civita symbol. Note that the wavenumber and the conductivity always enter through the combination $\tilde{\sigma}=\sigma / k$.

After straightforward algebra, the characteristic polynomial of (14) can be written as

$$
\mathcal{P}(\lambda)=\lambda \mathcal{P}_{5}(\lambda) \mathcal{P}_{4}(\lambda),
$$

where $\mathcal{P}_{5}(\lambda)$ and $\mathcal{P}_{4}(\lambda)$ are given by

$$
\begin{aligned}
\mathcal{P}_{5}(\lambda)= & \lambda^{5}+i \tilde{\sigma}\left(u_{A}^{2}+1\right) \lambda^{4}-\left(a^{2}+1\right) \lambda^{3} \\
& -i \tilde{\sigma}\left(a^{2} u_{A}^{2} \cos ^{2} \theta+a^{2}+u_{A}^{2}\right) \lambda^{2} \\
& +a^{2} \lambda+i \tilde{\sigma} a^{2} u_{A}^{2} \cos ^{2} \theta
\end{aligned}
$$

and

$$
\begin{aligned}
\mathcal{P}_{4}(\lambda)= & \lambda^{4}+i \tilde{\sigma}\left(u_{A}^{2}+2\right) \lambda^{3}-\left[\left(u_{A}^{2}+1\right) \sigma^{2}+1\right] \lambda^{2} \\
& -i \tilde{\sigma}\left(u_{A}^{2}+1\right) \lambda+\tilde{\sigma}^{2} u_{A}^{2} \cos ^{2} \theta .
\end{aligned}
$$

Note that $\mathcal{P}_{4}(\lambda)$ could have been directly obtained from the sub-matrix involving only the equations for $v_{z 1}, B_{z 1}, E_{x 1}$, and $E_{y 1}$ which are not coupled to the remaining variables.

Equations (18) and (19) have been expressed in terms of the four parameters $a^{2}, u_{A}^{2}, \theta$, and $\tilde{\sigma}$ which we now briefly describe.

- The first parameter, $a^{2}$, defines the square of the sound speed which can be defined in terms of the derivatives of the gas enthalpy $w$

$$
a^{2}=\frac{w_{0}-\rho_{0} w_{0, \rho}^{\prime}}{w_{0, p}^{\prime}-1} \frac{1}{w_{0}} .
$$

For an ideal gas, $w_{0}=\rho_{0}+\Gamma p_{0} /(\Gamma-1)$ so that the sound speed becomes $a=\sqrt{\Gamma p_{0} / w_{0}}$, where $\Gamma$ is the specific heat ratio. Note that $\mathcal{P}_{4}$ is independent of the sound speed.

- The second parameter is the magnetization $u_{A}^{2}=B_{0}^{2} / w$ $=v_{A}^{2} /\left(1-v_{A}^{2}\right)$, where

$$
v_{A}=\frac{\left|B_{0}\right|}{\sqrt{w_{0}+B_{0}^{2}}}
$$

reduces to the Alfvén velocity in the case of parallel propagation.

- The third parameter is the angle $\theta$ between the magnetic field and the wavevector

$$
\theta=\arctan \left(\frac{B_{0 y}}{B_{0 x}}\right) .
$$

- Finally, the fourth parameter is $\tilde{\sigma}=\sigma / k$.

The zeros of the characteristic polynomial give the desired dispersion relation. From Eq. (17), we immediately see that $\mathcal{P}(\lambda)$ possesses one trivial root $\lambda=0$, which corresponds to the contact (or entropy) mode. The other propagation modes are given by the roots $\mathcal{P}_{5}$ and $\mathcal{P}_{4}$.

While some general properties of the solution can be established by inspecting the two polynomials (Sec. III), the actual eigenmodes and their dependency on the parameters have to be investigated numerically (Sec. IV).

\section{GENERAL PROPERTIES OF THE SOLUTION}

In general, the eigenvalues $\lambda$ of the system are complex quantities and the real part identifies the phase velocity, i.e., $v_{p} \equiv \Re(\lambda)$ while the damping rate is proportional to the imaginary part through $-k \Im(\lambda)$.

By taking the complex conjugate of $\mathcal{P}_{5}$ or $\mathcal{P}_{4}$, it is easily seen that if $\lambda$ is a solution then the opposite of its complex conjugate, $-\bar{\lambda}$, is also a solution. Thus roots with a non-zero real part must always come as pairs of left- and right-going propagating waves with equal damping rates. Solution modes of this kind, with non-zero phase velocity, will be labeled type $P$ modes. In addition, as shown in Appendix A, $\mathcal{P}_{5}$ should always admit a strictly imaginary solution $(\Re(\lambda)=0)$ which corresponds to a purely damped, nonpropagating mode. Likewise, $\mathcal{P}_{4}$ always has (at least) two imaginary solutions. Solution modes of this kind will be labeled as type $N$ modes.

As we shall see, the system is dissipative since $-\Im(\lambda)>0$ and also dispersive since the phase velocity depends nonlinearly on $\tilde{\sigma}=\sigma / k$ and therefore on the wavenumber $k$. The group velocity can be calculated directly using

$$
v_{g} \equiv \frac{d \omega}{d k}=-\frac{d(\lambda / \tilde{\sigma})}{d \tilde{\sigma}} \tilde{\sigma}^{2} .
$$

Near degenerate points (roots with multiplicity two or higher), Eq. (23) can occasionally exceed unity and the 
system presents peculiarities of anomalous dispersion (regions where the group velocity becomes superluminal). This, however, does not violate causality as we discuss in Sec. IV D.

In Secs. III A and III B, we derive analytical expressions which hold in the limit of small $\tilde{\sigma}$ (the resistive limit) and large $\tilde{\sigma}$ (ideal limit). We point out that the resistive limit can be obtained by either fixing the wave number and letting $\sigma$ $\rightarrow 0$ or, alternatively, by fixing the conductivity and considering large wavenumbers. Conversely, the ideal limit is recovered for a large value of $\sigma$ (at fixed wavelength) or for small wavenumbers (at fixed $\sigma$ ).

\section{A. Resistive limit $(\tilde{\sigma} \rightarrow 0)$} fies to

In the $\tilde{\sigma} \rightarrow 0$ limit, one can easily show that $\mathcal{P}_{5}$ simpli-

$$
\mathcal{P}_{5}^{(\tilde{\sigma} \rightarrow 0)}=\lambda\left[\lambda^{4}-\left(a^{2}+1\right) \lambda^{2}+a^{2}\right]=0,
$$

whose solutions are

$$
\lambda_{1}=0 \quad \lambda_{2,3}= \pm a \quad \lambda_{4,5}= \pm 1
$$

The solutions are thus given by four propagation modes (a pair of acoustic waves and a pair of light modes) and a nonpropagating mode. This is not surprising since, for $\tilde{\sigma} \rightarrow 0$ (infinite resistivity limit), electromagnetic waves and fluid motion are no longer coupled.

Likewise, in the resistive limit, $\mathcal{P}_{4}$ reduces to

$$
\mathcal{P}_{4}^{(\tilde{\sigma} \rightarrow 0)}=\lambda^{2}\left(\lambda^{2}-1\right)=0
$$

with solutions

$$
\lambda_{6,7}=0 \quad \lambda_{8,9}= \pm 1
$$

representing a pair of type $N$ non-propagating modes and a pair of light waves.

Using a perturbative expansion in $\tilde{\sigma}$, we find that the first-order correction terms to the eigenvalues are, for the roots of $\mathcal{P}_{5}$,

$$
\begin{aligned}
& \lambda_{1} \approx-i \tilde{\sigma} u_{A}^{2} \cos ^{2} \theta+O\left(\tilde{\sigma}^{3}\right), \\
& \lambda_{2,3} \approx \pm a-i \frac{\tilde{\sigma}}{2} u_{A}^{2} \sin ^{2} \theta+O\left(\tilde{\sigma}^{2}\right), \\
& \lambda_{4,5} \approx \pm 1-i \frac{\tilde{\sigma}}{2}+O\left(\tilde{\sigma}^{2}\right),
\end{aligned}
$$

valid, of course, only for $\tilde{\sigma} \ll 1$. Similarly, we find for $\mathcal{P}_{4}$ the regular expansion

$$
\begin{aligned}
& \lambda_{6,7}=-i \frac{\tilde{\sigma}}{2}\left(u_{A}^{2}+1\right)\left[1 \pm \sqrt{1-\frac{4 u_{A}^{2} \cos ^{2} \theta}{\left(u_{A}^{2}+1\right)^{2}}}\right]+O\left(\tilde{\sigma}^{3}\right), \\
& \lambda_{8,9}= \pm 1-i \frac{\tilde{\sigma}}{2}+O\left(\tilde{\sigma}^{2}\right) .
\end{aligned}
$$

Note that, to the first-order in $\tilde{\sigma}$, the imaginary part of the light modes is $-\tilde{\sigma} / 2$, as also shown by Takamoto and Inoue $^{15}$ in the case of parallel propagation.

In our notations, $\lambda_{k}$ with $k=2,3,4,5,8,9$ are type $P$ modes, while $\lambda_{k}$ with $k=1,6,7$ are type $N$ modes. All roots have negative imaginary parts which indicate damping. The four light modes $\left(\lambda_{4,5}\right.$ and $\left.\lambda_{8,9}\right)$ behave essentially in the same way and the damping rate varies linearly with the conductivity and it does not depend on the sound speed. The damping rate of the acoustic wave is proportional to the magnetization and the inclination angle. The three type $N$ modes $\left(\lambda_{1}\right.$ and $\left.\lambda_{6,7}\right)$ have different damping rates, which all increase with the magnetization $\left(\propto B_{0}^{2}\right)$. For perpendicular propagation, two of them vanish identically and only one is non-zero. As we shall see later, this feature holds for any value of $\tilde{\sigma}$. Interestingly, it can be shown that the phase velocities of the type $P$ modes involve only even powers of $\tilde{\sigma}$, while the damping term can be expressed as a series of odd powers.

\section{B. Ideal limit $(\tilde{\sigma} \rightarrow \infty)$}

In the limit $\tilde{\sigma} \rightarrow \infty$, we have that $\mathcal{P}_{5}$ reduces to the following biquadratic equation:

$$
\begin{aligned}
\mathcal{P}_{5}^{(\tilde{\sigma} \rightarrow \infty)}= & \left(u_{A}^{2}+1\right) \lambda^{4}-\left(a^{2} u_{A}^{2} \cos ^{2} \theta+a^{2}+u_{A}^{2}\right) \lambda^{2} \\
& +a^{2} u_{A}^{2} \cos ^{2} \theta .
\end{aligned}
$$

Equation (30) admits four propagating modes given by the fast and slow magnetosonic speeds (see, e.g., Ref. 4)

$$
\begin{aligned}
& \lambda_{f \pm}= \pm \sqrt{\frac{a^{2} u_{A}^{2} \cos ^{2} \theta+a^{2}+u_{A}^{2}+\sqrt{\Delta}}{2\left(u_{A}^{2}+1\right)}}, \\
& \lambda_{s \pm}= \pm \sqrt{\frac{a^{2} u_{A}^{2} \cos ^{2} \theta+a^{2}+u_{A}^{2}-\sqrt{\Delta}}{2\left(u_{A}^{2}+1\right)}},
\end{aligned}
$$

where $\Delta=\left(a^{2} u_{A}^{2} \cos ^{2} \theta+a^{2}-u_{A}^{2}\right)^{2}+4 a^{2} u_{A}^{2} \sin ^{2} \theta$. Simple differentiation with respect to $\theta$ shows that $\lambda_{f \pm}$ and $\lambda_{s \pm}$ are, respectively, monotonically increasing and decreasing functions of $\theta$ in the range $\theta \in[0, \pi / 2]$. Therefore, one always has that $\lambda_{s, \pm}^{2} \leq a^{2} \leq \lambda_{f, \pm}^{2}$. The same condition holds in the non-relativistic limit which is easily obtained by letting $u_{A}^{2}$ $+1 \rightarrow 1$ and $a^{2} u_{A}^{2} \rightarrow 0$.

In the same limit, one finds that $\mathcal{P}_{4}$ reduces to the simple quadratic equation

$$
\mathcal{P}_{4}^{(\tilde{\sigma} \rightarrow \infty)}=\lambda^{2}\left(u_{A}^{2}+1\right)-u_{A}^{2} \cos ^{2} \theta=0,
$$

which admits a pair of Alfvén wave solutions

$$
\lambda_{A \pm}= \pm \frac{u_{A} \cos \theta}{\sqrt{u_{A}^{2}+1}} .
$$

The asymptotic behavior for large $\tilde{\sigma}$ can be obtained by conveniently introducing the resistivity parameter $\tilde{\eta}=1 / \tilde{\sigma}$ and rewriting Eqs. (18) and (19) as 


$$
\begin{aligned}
\mathcal{P}_{5}= & \tilde{\eta} \lambda^{5}+i\left(u_{A}^{2}+1\right) \lambda^{4}-\tilde{\eta}\left(a^{2}+1\right) \lambda^{3} \\
& -i\left(a^{2} u_{A}^{2} \cos ^{2} \theta+a^{2}+u_{A}^{2}\right) \lambda^{2} \\
& +a^{2} \lambda \tilde{\eta}+i a^{2} u_{A}^{2} \cos ^{2} \theta
\end{aligned}
$$

and

$$
\begin{aligned}
\mathcal{P}_{4}= & \tilde{\eta}^{2} \lambda^{4}+i \tilde{\eta}\left(u_{A}^{2}+2\right) \lambda^{3}-\left(\eta^{2}+u_{A}^{2}+1\right) \lambda^{2} \\
& -i \tilde{\eta}\left(u_{A}^{2}+1\right) \lambda+u_{A}^{2} \cos ^{2} \theta .
\end{aligned}
$$

Regular type $P$ solutions to these equations, in the limit $\tilde{\eta} \rightarrow 0(\tilde{\sigma} \rightarrow \infty)$, may be found using the same perturbative technique adopted in Sec. III A. The result is

$$
\begin{aligned}
& \lambda_{f \pm}(\tilde{\eta}) \approx \lambda_{f \pm}-i \frac{\tilde{\eta}}{2} \frac{\left(1-\lambda_{f \pm}^{2}\right)\left(\lambda_{f \pm}^{2}-a^{2}\right)}{\sqrt{\Delta}}, \\
& \lambda_{s \pm}(\tilde{\eta}) \approx \lambda_{s \pm}-i \frac{\tilde{\eta}}{2} \frac{\left(1-\lambda_{s \pm}^{2}\right)\left(a^{2}-\lambda_{s \pm}^{2}\right)}{\sqrt{\Delta}}, \\
& \lambda_{A \pm}(\tilde{\eta}) \approx \lambda_{A \pm}-i \frac{\tilde{\eta}}{2}\left(1-\lambda_{A \pm}^{2} \frac{u_{A}^{2}+2}{u_{A}^{2}+1}\right),
\end{aligned}
$$

where $\lambda_{f \pm}$ and $\lambda_{s \pm}$ are given by (31). Equation (37) shows that the damping rate of fast and slow modes is proportional to $\tilde{\eta} \equiv k \eta$ and, since $\lambda=\omega / k$, we get that the damping rate is proportional to $\eta k^{2}$, i.e., it has, as expected, a diffusive behavior.

Equations (34) and (35) also admit asymptotically singular solutions which disappear when $\tilde{\eta} \rightarrow 0$. The asymptotic behavior can be recovered by the rescaling method, i.e., by setting $z=\lambda / \tilde{\eta}$ which turns the singular perturbation problem into a regular one. Solving the regularized problem in $z$ using the perturbative approach and then rewriting the solution in the original variable $\lambda$ yields the three type $N$ roots in the asymptotically singular (as) limit

$$
\begin{aligned}
& \lambda_{a s, 1}=-i \frac{u_{A}^{2}+1}{\tilde{\eta}}+i \frac{a^{2} u_{A}^{2} \sin ^{2} \theta+1}{\left(u_{A}^{2}+1\right)^{2}} \tilde{\eta}+O\left(\tilde{\eta}^{3}\right), \\
& \lambda_{a s, 2}=-i \frac{u_{A}^{2}+1}{\tilde{\eta}}+i \frac{\cos ^{2} \theta}{\left(u_{A}^{2}+1\right)^{2}} \tilde{\eta}+O\left(\tilde{\eta}^{3}\right), \\
& \lambda_{a s, 3}=-\frac{i}{\tilde{\eta}}+i \sin ^{2} \theta \tilde{\eta}+O\left(\tilde{\eta}^{3}\right),
\end{aligned}
$$

where the first solution $\left(\lambda_{a s, 1}\right)$ is the singular root of $\mathcal{P}_{5}$, while the remaining two come from $\mathcal{P}_{4}$.

\section{Eigenvector structure}

From Eq. (13), we can obtain a formal expression for the eigenvectors in terms of the eigenvalue $\lambda$. A generic eigenvector component represents a perturbation that can be written as $V_{1}=\left|V_{A}\right| e^{i\left(k x-\omega_{R} t+\varphi\right)} e^{\omega_{I} t}$, where $V_{A} \in \mathbb{R}$ is the wave amplitude and $\varphi$ is the wave phase.

Whenever a plane wave carries a non-zero density perturbation (compressible mode), we assume $\rho_{1}=\epsilon e^{i(k x-\omega t)}$ and, after some algebra, we obtain

$$
\left(\begin{array}{c}
\rho_{1} \\
v_{1 x} \\
v_{1 y} \\
v_{1 z} \\
B_{1 y} \\
B_{1 z} \\
E_{1 x} \\
E_{1 y} \\
E_{1 z} \\
p_{1}
\end{array}\right)=\rho_{1}\left(\begin{array}{c}
1 \\
\frac{\lambda}{\rho_{0}} \\
\frac{\lambda \sin \theta \cos \theta \Delta_{2}}{\rho_{0} \Delta} \\
0 \\
-\frac{\lambda^{2} w_{0} B_{0} \tilde{\sigma} \sin \theta}{\rho_{0} \Delta} \\
0 \\
0 \\
0 \\
\frac{\lambda^{3} w_{0} B_{0} \tilde{\sigma} \sin \theta}{\rho_{0} \Delta} \\
\frac{\lambda^{2} w_{0}\left(\Delta_{1}+\Delta_{2}\right)}{\rho_{0} \Delta}
\end{array}\right),
$$

where $\quad \Delta=\Delta_{1}+\cos ^{2} \theta \Delta_{2}, \Delta_{1}=\lambda w_{0}\left(i \lambda^{2}-\lambda \tilde{\sigma}-i\right), \Delta_{2}$ $=\tilde{\sigma} B_{0}^{2}\left(1-\lambda^{2}\right)$. Compressible modes are possible only if $\lambda$ is a root of $\mathcal{P}_{5}$ [roots of $\mathcal{P}_{4}$ do not involve density perturbations as explained after Eq. (19)]. From the previous expression, it is seen that velocity and magnetic field perturbations lie in the plane defined by $\boldsymbol{k}$ and $\boldsymbol{B}_{0}$, whereas the electric field is orthogonal to this plane. In the infinite conductivity limit, perturbations are real quantities and the resulting expressions are well-behaved yielding the eigenvectors for the fast and slow magnetosonic waves (see Appendix B). For finite values of $\tilde{\sigma}$, perturbations become complex quantities and a phase shift appears. Of particular interest is the case of a purely imaginary eigenvalue, i.e., $\lambda=i Y$ : Eq. (38) shows that velocity and electric field perturbations become out of phase by $\pi / 2$ with respect to those of density, magnetic field, and pressure.

By setting $\rho_{1}=0$ in Eq. (13), only the $4 \times 4$ sub-system formed by the equations of $\left\{v_{1 z}, B_{1 z}, E_{1 x}, E_{1 y}\right\}$ has a nontrivial solution. The incompressible perturbation modes are thus associated with the roots of $\mathcal{P}_{4}$ and can be written as

$$
\left(\begin{array}{c}
\rho_{1} \\
v_{1 x} \\
v_{1 y} \\
v_{1 z} \\
B_{1 y} \\
B_{1 z} \\
E_{1 x} \\
E_{1 y} \\
E_{1 z} \\
p_{1}
\end{array}\right)=B_{1 z}\left(\begin{array}{c}
0 \\
0 \\
0 \\
\frac{1-\lambda^{2}-i \lambda \tilde{\sigma}}{i \tilde{\sigma} B_{0} \cos \theta} \\
0 \\
1 \\
\tan \theta \frac{1-\lambda^{2}-i \lambda \tilde{\sigma}}{\lambda+i \tilde{\sigma}} \\
\lambda \\
0 \\
0
\end{array}\right) .
$$

Modes described by Eq. (39) propagate fluctuations of velocity and electromagnetic field components perpendicular to the plane defined by $\boldsymbol{k}$ and $\boldsymbol{B}_{0}$.

Limit expressions in the resistive and ideal regimes are reported in Appendix B. 


\section{RESULTS}

We now study in detail the solutions of the characteristic polynomial by exploring the parameter space defined by $a$, $v_{A}, \theta$, and $\tilde{\sigma}$.

Since neither $\mathcal{P}_{5}$ nor $\mathcal{P}_{4}$ have simple analytical solutions for a finite value of the conductivity $\tilde{\sigma} \equiv \sigma / k$, we adopt a numerical approach based on the Durand-Kerner method ${ }^{20}$ which is widely used for calculating both the real and the complex roots of a univariate polynomial at the same time. Given a polynomial of $m$-th degree, the Durand-Kerner algorithm iterates on all of the roots $\lambda_{i}$ (with $i=1, \ldots, m$ ) simultaneously

$$
\lambda_{i}^{(k+1)}=\lambda_{i}^{(k)}-\frac{\mathcal{P}_{m}\left(\lambda_{i}^{(k)}\right)}{\prod_{j \neq i}\left(\lambda_{i}^{(k)}-\lambda_{j}^{(*)}\right)},
$$

where $k$ is the iteration cycle and $\lambda_{j}^{*}$ is the most recent updated value $\left(\lambda^{(*)}=\lambda_{j}^{(k)}\right.$ if $j>i$ or $\lambda^{(*)}=\lambda_{j}^{(k+1)}$ otherwise). The iteration process converges quadratically provided sufficiently close guesses are provided.

Equation (40) is typically solved by fixing $a, u_{A}$, and $\theta$ for different values of the conductivity $\tilde{\sigma}$. We start at $\tilde{\sigma}=0$ where we have exact expressions for the eigenvalues given by Eqs. (25) and (27), respectively. These values are then used as guesses to start the iteration cycle for the next value of $\tilde{\sigma}$.

We first discuss, in Secs. IV A and IV B, the characteristic modes of $\mathcal{P}_{5}$ and $\mathcal{P}_{4}$ for fixed orientation angle $\theta=0.7$ $\approx 40^{\circ}$ ). Next, in Sec. IV C, we examine the behavior of the system at arbitrary angles $\theta$.

As already stated in Sec. III, we conveniently label type $P$ mode pairs of propagating waves with non-zero phase velocity, that is, $\lambda^{(P)}= \pm \Re(\lambda)+i \Im(\lambda)$. On the contrary, type $N$ modes are purely imaginary, nonpropagating damped modes and have the form $\lambda^{(N)}=i \Im(\lambda)$. A transition from a type $P$ mode to a type $N$ mode (e.g., light to purely damped waves) can occur through a degeneracy point characterized by a root of multiplicity two. In these cases, degeneracy points are (by convention) named after the limiting value of the type $P$ mode at $\tilde{\sigma} \rightarrow 0$ (for a $P-N$ transition) or $\tilde{\sigma} \rightarrow \infty$ (for an $N-P$ transition). Likewise, a pair of degeneracy points appears in correspondence of two double roots and marks a transition between pairs of type $P$ modes (e.g., lightacoustic).

\section{A. Mode analysis for $\mathcal{P}_{5}$}

We first consider the eigenvalues of $\mathcal{P}_{5}$ in the cold $(a=0.15)$ and hot $(a=0.55)$ gas cases and study the behavior of the system for different values of the magnetization.

\section{Low magnetization $\left(0.1 \leqq v_{A} \lesssim 0.2\right)$ - cold gas}

In Fig. 1, we plot the roots of $\mathcal{P}_{5}$ for $v_{A}=0.1$ and $\theta$ $=0.7$. In the left panel, the real and imaginary parts are plotted as functions of $\tilde{\sigma}=\sigma / k$, while the right panel gives the path followed in the complex $\lambda$ plane. The different curves show the five modes which can be easily identified in the limit of zero conductivity [see Eq. (25)]. Starting at $\tilde{\sigma}=0$, in fact, we have a pair of light modes $\lambda_{2,3}= \pm 1$ (red and orange curves in the figure), a pair of acoustic modes $\lambda_{4,5}= \pm a$ (blue and cyan), and a null-mode $\lambda_{1}=0$ (black). In the limit of small $\tilde{\sigma}$, our results agree with the expansion given in Eq. (28).

For $0 \leqq \tilde{\sigma} \lesssim 1.96$, the phase velocities of the light modes decrease (in absolute value) until they become degenerate reaching zero phase speed. The light degeneracy point sets the transition to a pair of type $N$ modes and the corresponding formation of a pair of damped standing waves for $1.96 \leqq \tilde{\sigma} \lesssim 6$ (red and orange curves on the imaginary axis in the left panel of Fig. 1). As noticed in Sec. III C, modes with a purely imaginary part are characterized by a $\pi / 2$ phase shift between velocity and magnetic field perturbations. The
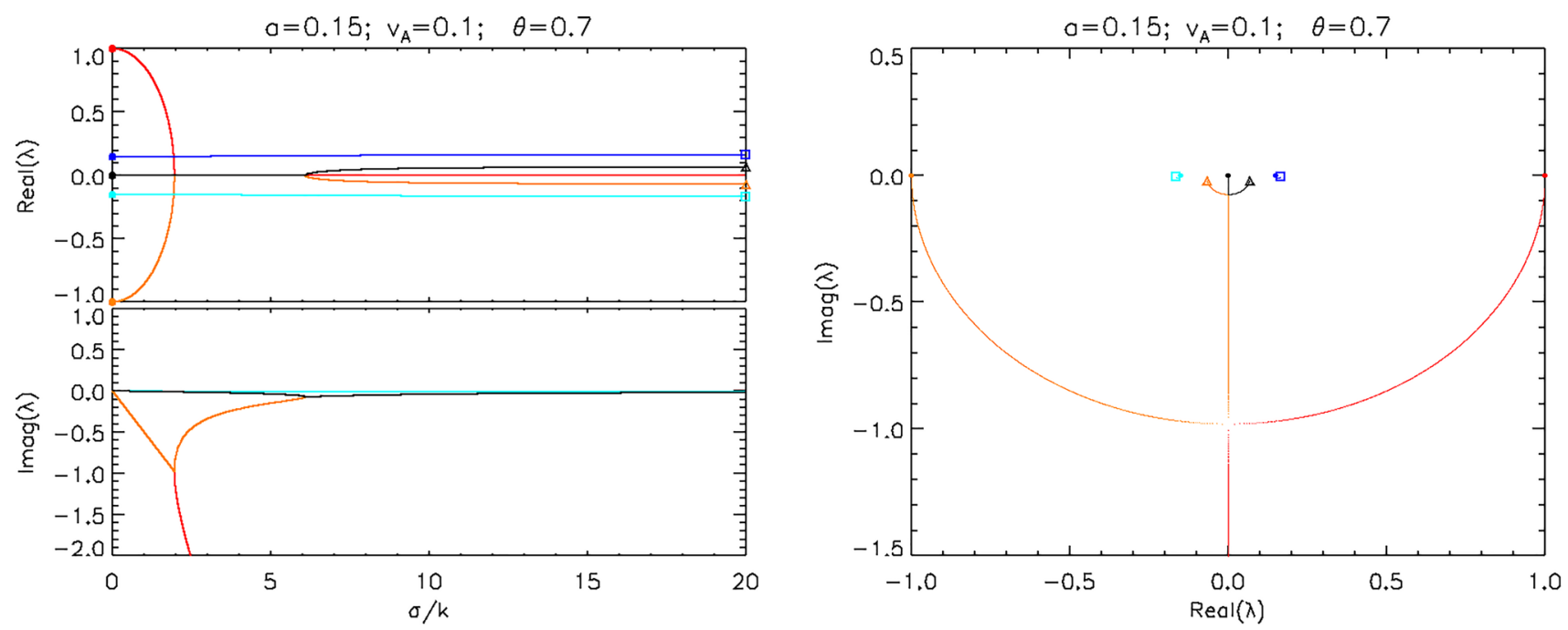

FIG. 1. Roots of $\mathcal{P}_{5}$ in the cold gas case $(a=0.15)$, low magnetization regime $\left(v_{A}=0.1\right)$ and $\theta=0.7$. In the left panel, we plot the real and imaginary parts of the solution as functions of $\tilde{\sigma} \equiv \sigma / k$. The right panels show the corresponding eigenmode positions in the complex $\lambda$ plane (initial values at $\tilde{\sigma}=0$ are denoted with small filled circles). At small values of $\tilde{\sigma}$, blue and cyan curves denote the acoustic modes while red and orange curves represent the light modes; the black line is a purely damped mode. At large values of $\tilde{\sigma}$, blue and cyan curves tend to the fast magnetosonic waves (small squares), black and orange curves approach the slow magnetosonic waves (small triangles), while the red line show the rapidly damped mode. 
damping rates of the type $N$ modes have an opposite trend: while one of the two modes becomes rapidly suppressed (red), the other one (orange) features a decreasing damping rate until it merges with the purely damped mode (black) at $\tilde{\sigma} \approx 6$. This settles the slow degeneracy point and the transition to type $P$ modes which asymptotically approach a pair of left- and right-going slow magnetosonic waves.

The acoustic modes (blue and cyan), on the other hand, remain always distinct and are characterized by very small damping rates which vanishes as they approach the fast magnetosonic speed in the ideal limit, see Eq. (28). They also weakly depend on $\tilde{\sigma}$.

\section{Moderate magnetization $\left(v_{A} \approx 0.19\right)$ - cold gas}

By increasing the magnetization, the light and acoustic modes move closer in the complex plane. At $v_{A} \approx 0.193$, two double roots appear (the light-acoustic degeneracy point) and hence, the two mode pairs switch their asymptotic branches: the acoustic modes now tend to the slow magnetosonic waves (rather than the fast), while the light modes approach the fast (rather than the slow) modes. This pattern is best illustrated in Fig. 2 where the roots are plotted in the complex $\lambda$ plane immediately prior and after the degeneracy, which takes place for $\tilde{\sigma} \approx 3.8$.

\section{High magnetization (0.25 $\left.\leqslant v_{A} \leq 0.41\right)$ - cold gas}

For $v_{A}=0.25$ (top panels in Fig. 3), the light degeneracy point shifts at a slightly smaller value of $\tilde{\sigma} \approx 1.75$. Damped standing waves (corresponding to a pair of type $N$ modes) form in a much narrower range on the negative imaginary axis. At $\tilde{\sigma} \approx 2.1$, we have again a type $N$-type $P$ transition through the fast degeneracy point leading to a pair of forward/reverse waves approaching the fast magnetosonic speed (rather than the slow) in the $\tilde{\sigma} \rightarrow \infty$ limit.

When the magnetization is further increased to $v_{A}$ $=0.41$ (bottom panels), degeneracies are removed and all roots remain distinct for any value of $\tilde{\sigma}$. This is best seen in the bottom right panel of Fig. 3 where four type $P$ modes (orange, cyan, blue, and red) and an isolated type $N$ solution are visible. While the acoustic modes smoothly connect with the slow mode in the ideal limit, the phase velocities of the light waves decrease, in absolute value, to a minimum (found at $\sigma \approx 1.43$ ) and shortly after rapidly approach the fast magnetosonic speeds. Finally, the type $N$ mode increases linearly for $\tilde{\sigma} \lesssim 1$ [see the first equation in (28)] and then much faster for $\tilde{\sigma} \gtrsim 1$.

\section{Results for a hot gas}

Next we increase the sound speed to $a=0.55$ (slightly below the asymptotic value $1 / \sqrt{3}$ ), in order to investigate relativistic thermodynamic effects. Eigenvalues are plotted in the six panels of Fig. 4 for increasing values of the magnetization (from top to bottom, $v_{A}=0.25,0.45$, and 0.6 , respectively). Although the qualitative behavior is essentially the same one identified for the cold gas case, few differences are discernible.

For $v_{A} \lesssim 0.25$ (top panels), we again have, for increasing $\tilde{\sigma}$, two light waves followed by a pair of type $N$ modes and then a pair of slow magnetosonic waves. The damped standing waves are delimited by the two degeneracy points around $\tilde{\sigma} \approx 1.77$ and $\tilde{\sigma} \approx 2.48$. Acoustic modes (blue and cyan) show a weak dependence of the conductivity and smoothly connect to the fast magnetosonic waves.

At $v_{A}=0.45$ (middle panels), degeneracies have been removed and we have again five distinct modes (4 type $P$ solutions and 1 type $N$ mode). Light and slow magnetosonic waves are connected continuously and so are the acousticfast magnetosonic waves. The non-propagating type $N$ mode (black) becomes quickly damped as $\tilde{\sigma}$ increases.

Finally, when the magnetization reaches $v_{A}=0.6$ (bottom panels), light and acoustic modes swap their asymptotic behavior through a double degeneracy point: the light (acoustic) modes approach the fast (slow) magnetosonic speeds. The type $N$ mode shows the same features as in the cold gas case as its asymptotic behavior [see $\lambda_{a s, 1}$ in Eq. (37)] is independent of the sound speed.

\section{B. Mode analysis for $\mathcal{P}_{4}$}

Since $\mathcal{P}_{4}$ does not depend on the sound speed, it suffices to consider different values of $v_{A}$. The overall behavior of roots is qualitatively similar albeit simpler than the cases discussed above. This is shown in the two panels of Fig. 5 for $v_{A}=0.25$ (top) and $v_{A}=0.6$ (bottom). For small values of $\tilde{\sigma}$, we always have two damped light modes (red and orange curves) and a pair of purely damped type $N$ modes with different imaginary parts (blue and cyan lines).
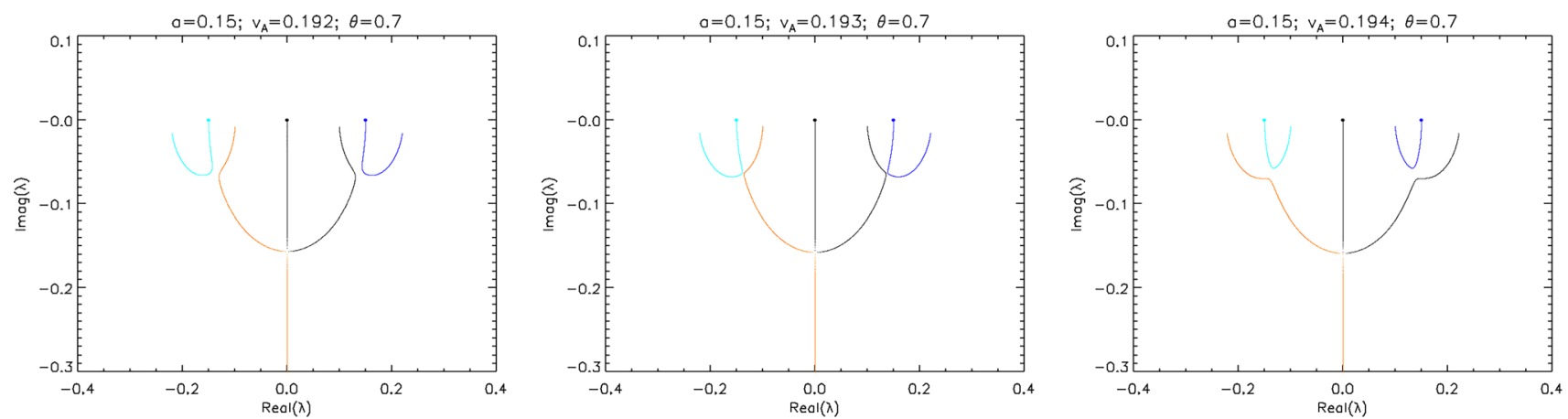

FIG. 2. Merging and asymptotic switch of the light and acoustic modes in the complex plane. From left to right, the three panels trace the eigenmode position in the complex plane for $v_{A}=0.191,0.192$, and 0.193 . The double degeneracy point takes place in the middle panel. 

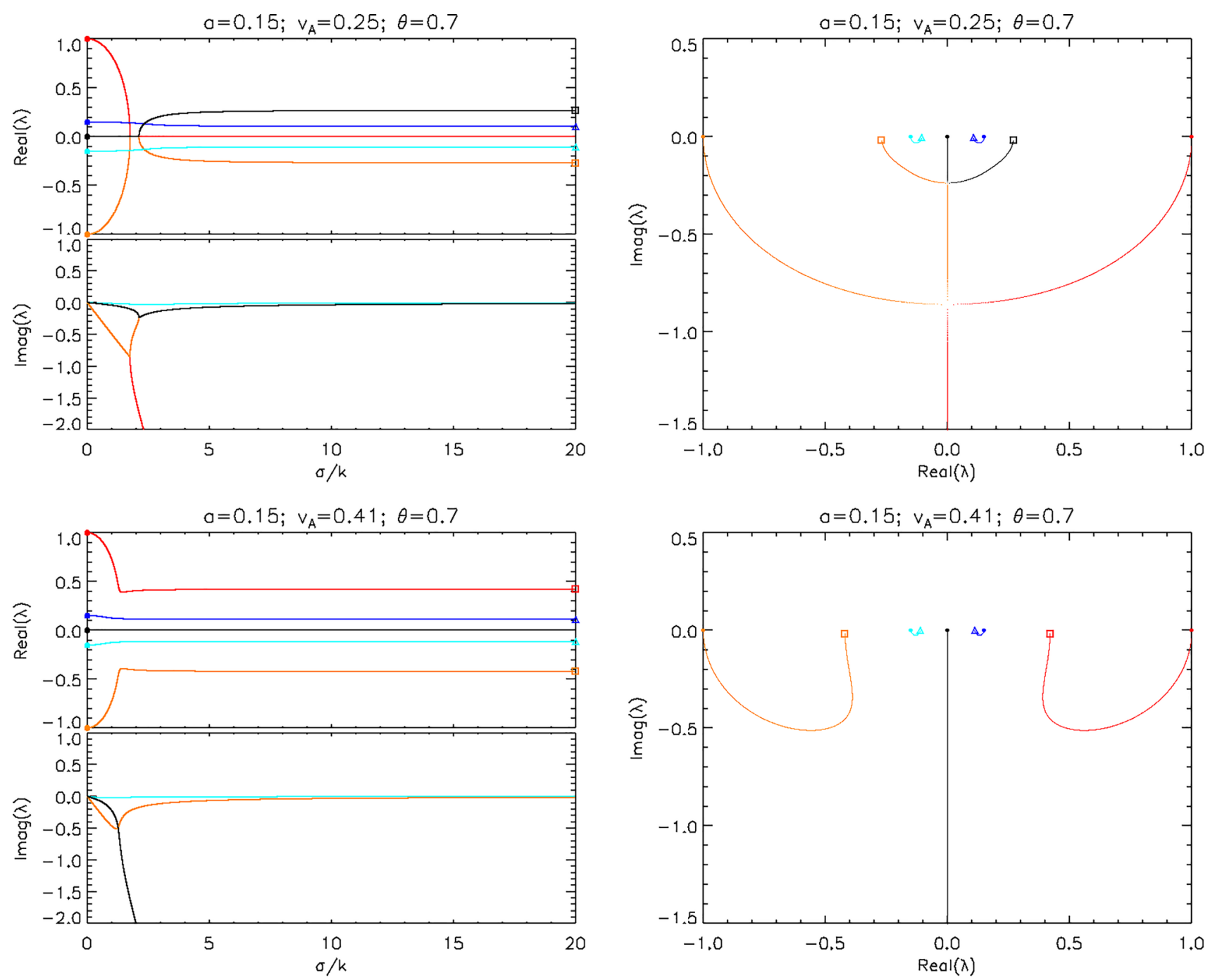

FIG. 3. Roots of $\mathcal{P}_{5}$ in the cold gas case $(a=0.15)$ for larger magnetizations corresponding to $v_{A}=0.25$ (top panel) and $v_{A}=0.41$ (bottom panel). Plot symbols have the same meaning as in Fig. 1 .

To first-order in $\tilde{\sigma}$, these modes are given by the regular expansions in Eq. (29). The mode with larger damping (cyan) remains always distinct and it coincides with $\lambda_{7}$ in Eq. (29) or $\lambda_{a s, 3}$ in Eq. (37) in the small or large $\tilde{\sigma}$ limits, respectively.

For $v_{A}=0.25$, the phase velocity of the light modes decreases (in absolute value) and a type P-type $N$ transition takes place at the light degeneracy point around $\sigma \approx 1.86$ (top panels). Here, the imaginary part of the light modes is intermediate between the two damped modes, i.e., $\Im\left(\lambda_{6}\right)<\Im\left(\lambda_{8,9}\right)<\Im\left(\lambda_{7}\right)$. A pair of damped standing waves forms for a narrow value range of $\tilde{\sigma}(1.86 \leqq \tilde{\sigma} \lesssim 2.62)$ and while one of the two modes becomes rapidly suppressed, the other one (orange) features a smaller damping rate. At $\tilde{\sigma} \approx 2.62$, we have a second degeneracy (the Alfvén degeneracy point) accompanied by a type $N$-type $P$ mode transition. Increasing $\tilde{\sigma}$ leads to the appearance of Alfvén waves.

For $v_{A}=0.6$, both degeneracies have been removed and all roots are now distinct: a pair of smoothly connected lightAlfvén modes and a pair of damped modes with rapidly growing damping rates (bottom panels in Fig. 5). The two light modes decrease their speed of propagation until a minimum value in the range $1<\sigma<2$, and then approach the Alfvén velocity as $\sigma \rightarrow \infty$. In the same limit, the asymptotic expression for the type $N$ modes is given by the singular perturbation solution given in Eq. (37).

\section{Dependency on the angle $\theta$}

While in Secs. IV A and IV B the angle between the wavevector $\boldsymbol{k}$ and the magnetic field $\boldsymbol{B}$ has been fixed to $\theta$ $=0.7$, we now explore the effect of different orientation angles. We first consider, in Secs. IV C 1 and IV C 2, the limiting cases corresponding to parallel and perpendicular propagation and leave the discussion at arbitrary angles to Sec. IV C 3 .

\section{Parallel propagation $(\theta=0)$}

When $\boldsymbol{B}$ and $\boldsymbol{k}$ are aligned, the two characteristic polynomials simplify to

$$
\mathcal{P}_{5}^{\|}=\left(\lambda^{2}-a^{2}\right)\left[\lambda^{3}+i \tilde{\sigma}\left(1+u_{A}^{2}\right) \lambda^{2}-\lambda-i \tilde{\sigma} u_{A}^{2}\right],
$$



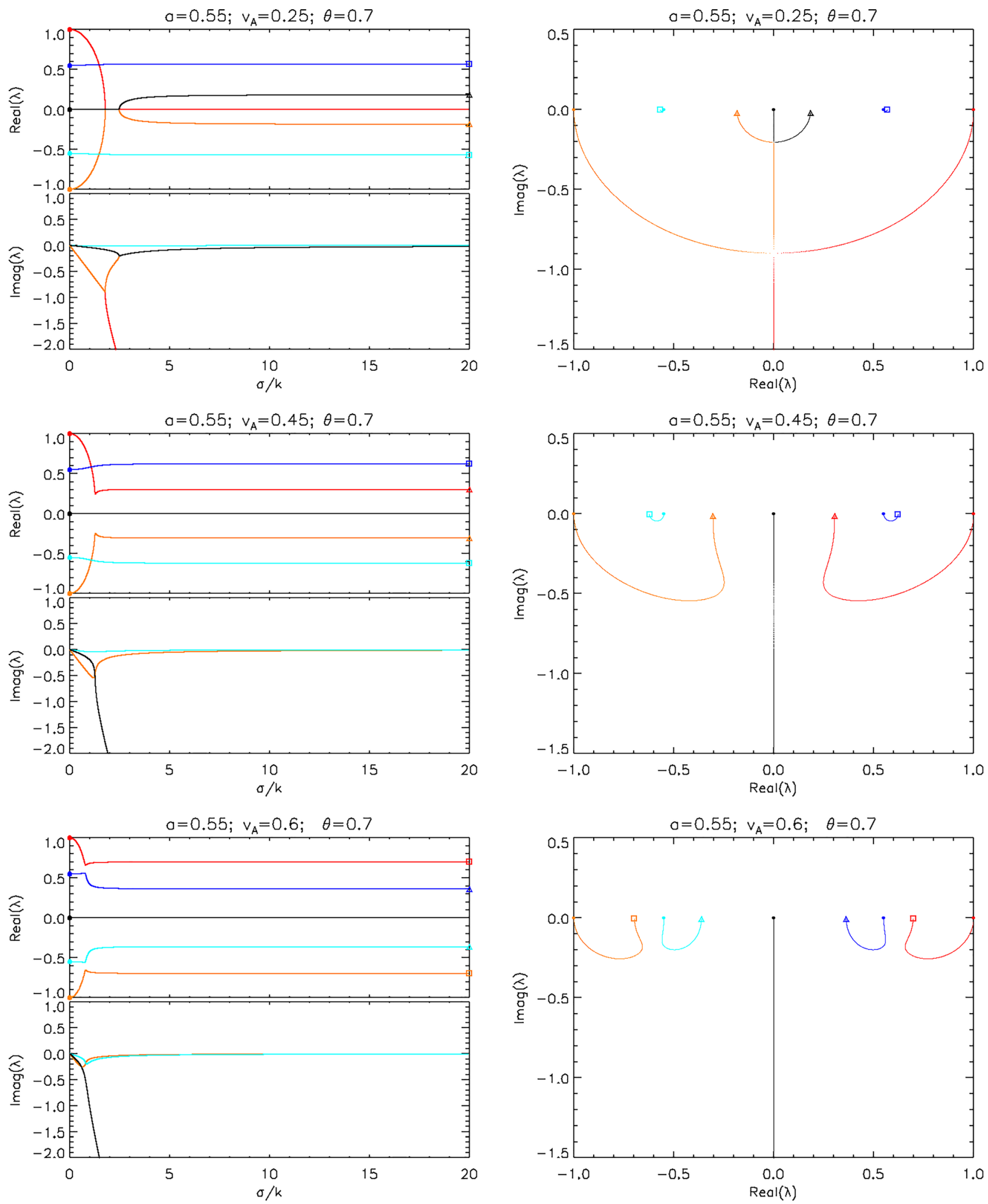

FIG. 4. Roots of $\mathcal{P}_{5}$ in the hot gas case $(a=0.55)$ and different magnetizations, as reported in the title. Plot symbols have the same meaning as in Fig. 1.

$$
\mathcal{P}_{4}^{\|}=(\lambda+i \tilde{\sigma})\left[\lambda^{3}+i \tilde{\sigma}\left(1+u_{A}^{2}\right) \lambda^{2}-\lambda-i \tilde{\sigma} u_{A}^{2}\right] .
$$

Equation (41) always admits the solutions $\lambda= \pm a$ which show that acoustic wave propagation is unaffected by electrical resistivity. Equation (42) has the solution $\lambda=-i \tilde{\sigma}$ which corresponds to the rapidly damped mode (again $\lambda_{7}$ or $\lambda_{s, 3}$ in the opposite limits). The remaining solutions are given by the roots of the cubic in square brackets which are common to both $\mathcal{P}_{5}$ and $\mathcal{P}_{4}$ and depend solely on $u_{A}$. They reduce to a null mode and a pair of light modes $\lambda= \pm 1,0$ (for $\tilde{\sigma} \rightarrow 0$ ) or a pair of Alfvén waves $\lambda= \pm v_{A}($ for $\tilde{\sigma} \rightarrow \infty)$. This result has also been found in the Appendix of Ref. 15 . 

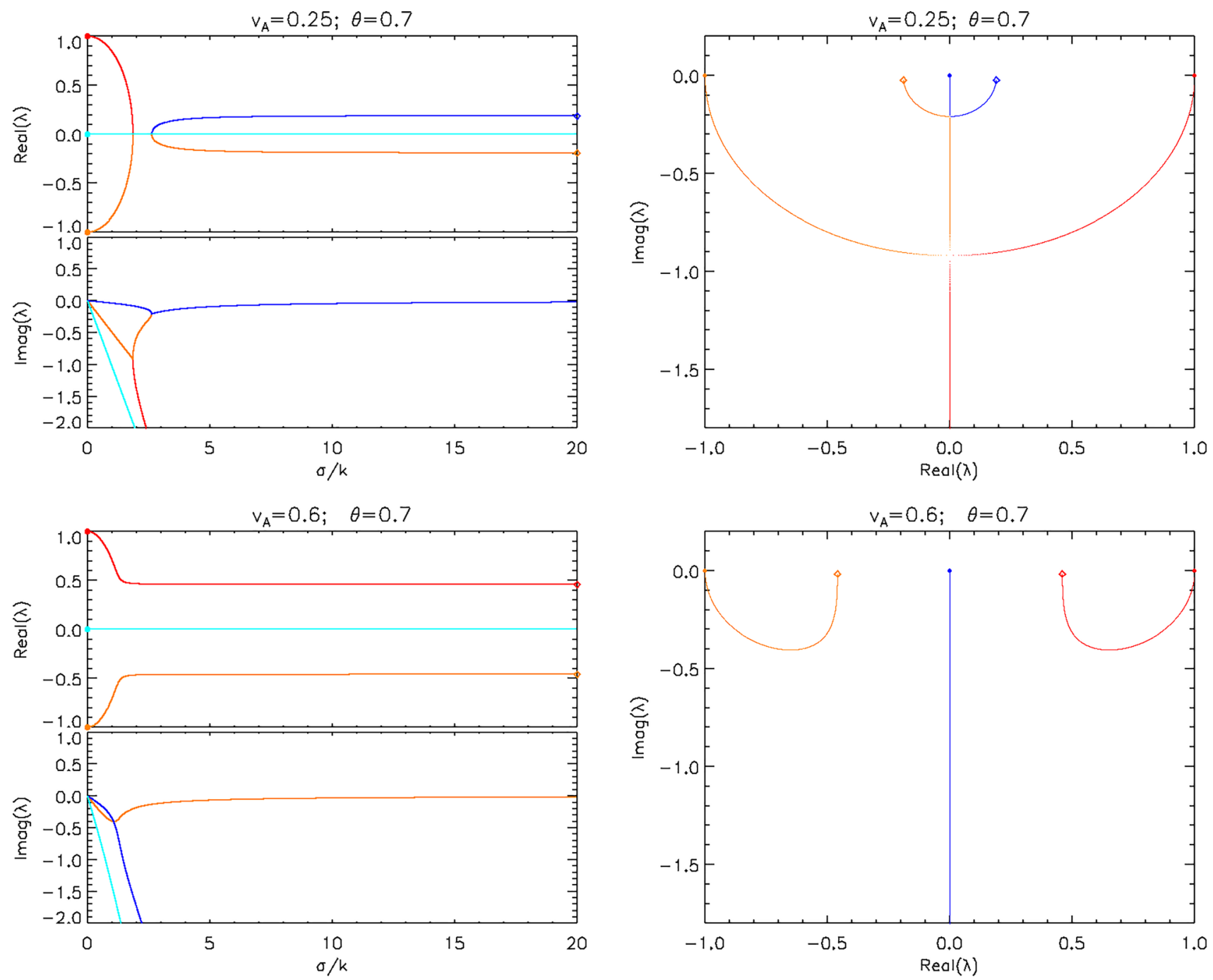

FIG. 5. Roots of $\mathcal{P}_{4}$ for $\theta=0.7$ and $v_{A}=0.25$ (top panels) or $v_{A}=0.6$ (bottom panels). The rapidly damped mode (cyan) has been omitted from the right panel for the sake of clarity.

From the discriminant of the cubic, it is easily found that a pair of type $N$ waves joining the light and Alfvén degeneracy points (given the black line segment with a vanishing real part in the left panel of Fig. 6) is found between the two values of $\tilde{\sigma}$ satisfying

$$
\tilde{\sigma}_{c}^{2}=\frac{-8 u_{A}^{4}+20 u_{A}^{2}+1 \pm\left(1-8 u_{A}^{2}\right)^{3 / 2}}{8 u_{A}^{2}\left(u_{A}^{2}+1\right)^{3}} .
$$

When $u_{A}=1 / \sqrt{8}\left(v_{A}=1 / 3\right)$, a triple root $\lambda=-i / \sqrt{3}$ forms at $\tilde{\sigma}_{c} \equiv 8 \sqrt{3} / 9 \approx 1.54$. The degeneracy is then removed when $v_{A} \geq 1 / 3$ so that five distinct roots appear with the two light modes always approaching the Alfvén velocity while the non-propagating mode becomes rapidly damped. This behavior, shown in the middle and right panels of Fig. 6, is also found in classical MHD.

\section{Perpendicular propagation $(\theta=\pi / 2)$}

When $\boldsymbol{k}$ and $\boldsymbol{B}$ are perpendicular, $\mathcal{P}_{5}$ reduces to the following expression:
$\mathcal{P}_{5}^{\perp}=\lambda\left[\lambda^{4}+i \tilde{\sigma}\left(u_{A}^{2}+1\right) \lambda^{3}-\left(a^{2}+1\right) \lambda^{2}-i \tilde{\sigma}\left(a^{2}+u_{A}^{2}\right) \lambda+a^{2}\right]$

which always has a vanishing root. At $\tilde{\sigma}=0$, we recover the usual pairs of light and acoustic modes while, in the limit $\tilde{\sigma} \rightarrow \infty$, the polynomial inside the square bracket admits the magnetoacoustic wave solution

$$
\lambda_{f \pm}= \pm \sqrt{\frac{a^{2}+u_{A}^{2}}{u_{A}^{2}+1}}
$$

and a second $\lambda=0$ solution. The two vanishing roots at $\tilde{\sigma}=\infty$ show that the slow magnetosonic modes disappear, as in classical MHD.

It is possible to show (see Appendix C) that the quartic inside the square brackets in Eq. (44) admits a triple root when

$$
v_{A, \pm}=\frac{\sqrt{2}}{4} \sqrt{\frac{B \pm\left(1-a^{2}\right) C^{3 / 2}}{\left(a^{2}+1\right)^{3}}} \quad \text { for } a<3-\sqrt{8},
$$



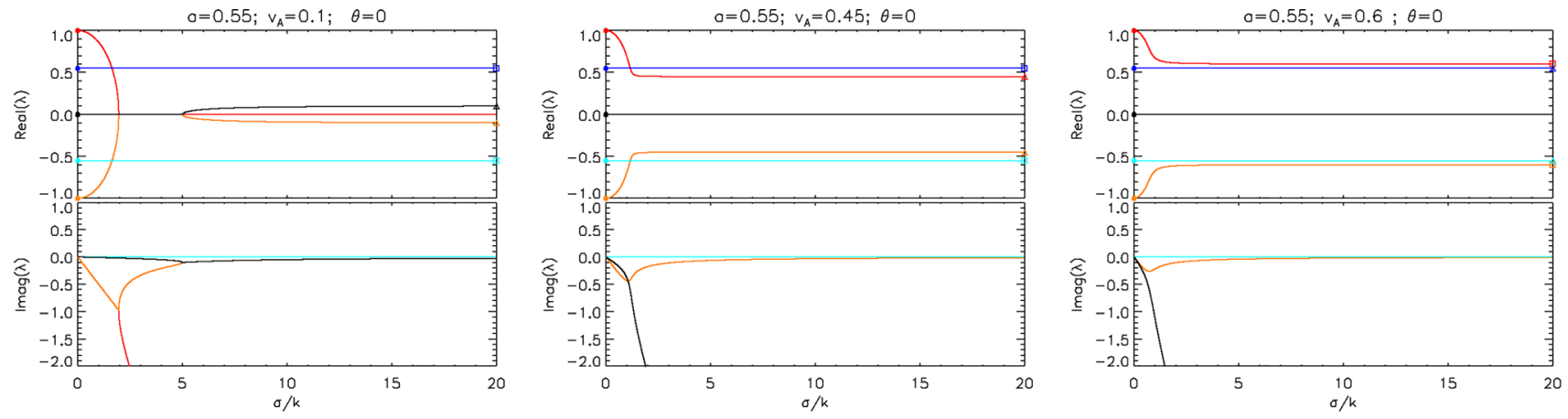

FIG. 6. Eigenmodes of $\mathcal{P}_{5}$ and $\mathcal{P}_{4}$ in the case of parallel propagation $(\theta=0)$ as a function of $\tilde{\sigma}$. Note that while the acoustic modes (blue and cyan curves) are roots of $\mathcal{P}_{5}$ only, the other modes (red, orange and black) are common roots to both $\mathcal{P}_{5}$ and $\mathcal{P}_{4}$. The non-propagating and rapidly damped mode of $\mathcal{P}_{4}$ has been omitted for clarity.

in correspondence of $\tilde{\sigma}$ given by Eq. (C4). The coefficients $B$ and $C$ are given immediately after Eq. (C6). In the $\left(a, v_{A}\right)$ plane (see Fig. 7), the two solutions given by Eq. (46) define the lower boundary curve above which light modes are no longer degenerate (for $v_{A}>v_{A,+}$ ) or the curve below which acoustic modes never degenerate (for $v_{A}<v_{A,-}$ ).

Also, a couple of double roots with non-zero phase speed appears when

$$
v_{A}=\sqrt{\frac{a}{1+a}}\left(\text { or } \quad u_{A}=\sqrt{a}\right), \quad \text { for } 0 \leq a \leq 1,
$$

in correspondence of $\tilde{\sigma}=2(1-a) /(1+a)$, where

$$
\lambda_{ \pm}^{\perp}=\frac{1}{2}\left[ \pm \sqrt{-a^{2}+6 a-1}-i(1-a)\right] .
$$

This pair of roots with multiplicity 2 marks a light-acoustic degeneracy point with the corresponding asymptotic switch (similar to the situation illustrated in Fig. 2).

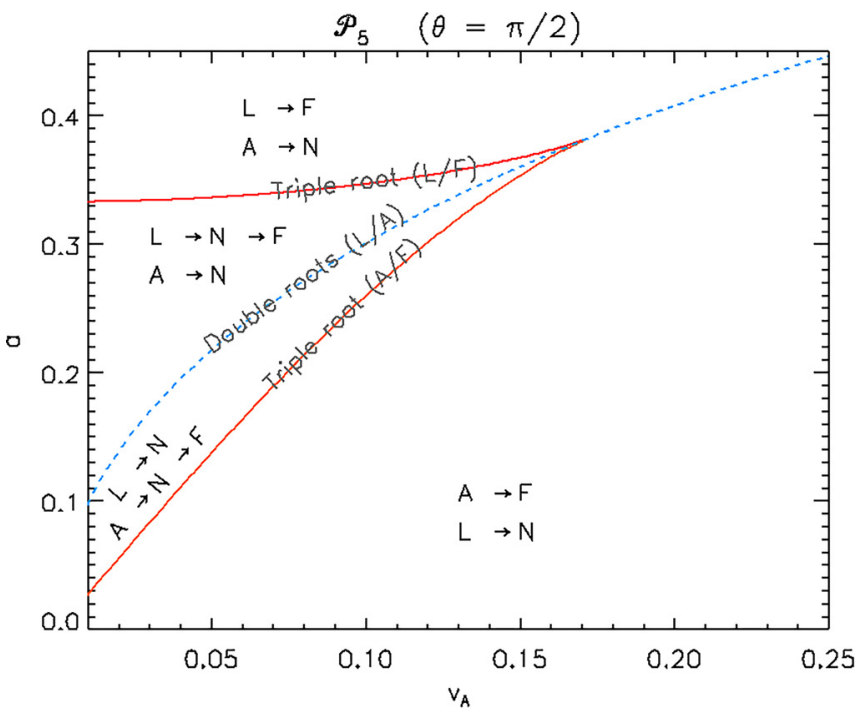

FIG. 7. Eigenmode degeneracies for $\mathcal{P}_{5}$ when $\theta=\pi / 2$. Red and orange curves give the locus of $\left(v_{A}, a\right)$ points where a triple root exist [plus and minus sign in Eq. (46)]. Light (acoustic) waves are never degenerate above (below) the red (orange) curve and they smoothly connect to the fast modes in the ideal limit. In-between the triple point curves, light (acoustic) waves become degenerate for a finite value range of $\tilde{\sigma}$ if they lie above (below) the blue line [Eq. (48)] but retain the same asymptotic limit.
Mode diagrams for different magnetizations $v_{A}=0.25$, 0.41 (cold gas) and $v_{A}=0.45, v_{A}=0.6$ (hot gas) are illustrated in Fig. 8. At small magnetizations (left panels), acoustic modes smoothly connect to the magnetoacoustic solution (45), while light waves transition to a pair of type $N$ modes. At large magnetizations (right panels), light and acoustic modes reverse their asymptotic behaviors: the light degeneracy point disappears being replaced by the acoustic degeneracy point through which acoustic waves transition to a pair of type $N$ modes. By increasing $\tilde{\sigma}$, one of these modes coincides with the rapidly damped mode (blue), while the second one (cyan) vanishes in the ideal limit.

The other four modes are given by the roots of

$\mathcal{P}_{4}^{\perp}=\lambda\left[\lambda^{3}+i \tilde{\sigma}\left(u_{A}^{2}+2\right) \lambda^{2}-\left[\left(u_{A}^{2}+1\right) \tilde{\sigma}^{2}+1\right] \lambda-i \sigma\left(u_{A}^{2}+1\right)\right]$,

which have the simple analytical expressions

$$
\lambda=\left\{\begin{array}{l}
0 \\
\frac{1}{2}\left(-i \tilde{\sigma} \pm \sqrt{4-\tilde{\sigma}^{2}}\right) \\
-i \tilde{\sigma}\left(u_{A}^{2}+1\right) .
\end{array}\right.
$$

In this case, there is a single light degeneracy (a root of multiplicity 2) always at $\tilde{\sigma}=2$ and it is independent of the magnetization. A triple root is not physically admissible in this case. The purely damped mode grows proportionally to $u_{A}^{2}$.

\section{Propagation at arbitrary angle $\theta$}

Taking advantage of the previous results, we now explore the behavior at intermediate values of $\theta$. The left panels in Fig. 9 show the locations of the degenerate roots for $\mathcal{P}_{5}$ for cold and hot gases (top and bottom plots on the left, respectively) in the $\left(\tilde{\sigma}, v_{A}\right)$ plane for different values of $\theta$ (corresponding to different colored curves).

Inside each curve, a pair of type $N$ modes exist; outside of this region, all roots (except the purely damped mode) are type $P$ modes. Across the leftmost branch of the curve, a root of multiplicity 2 sets the transition from type $P$ to type $N$, typically a light or acoustic mode degeneracy. Across the 

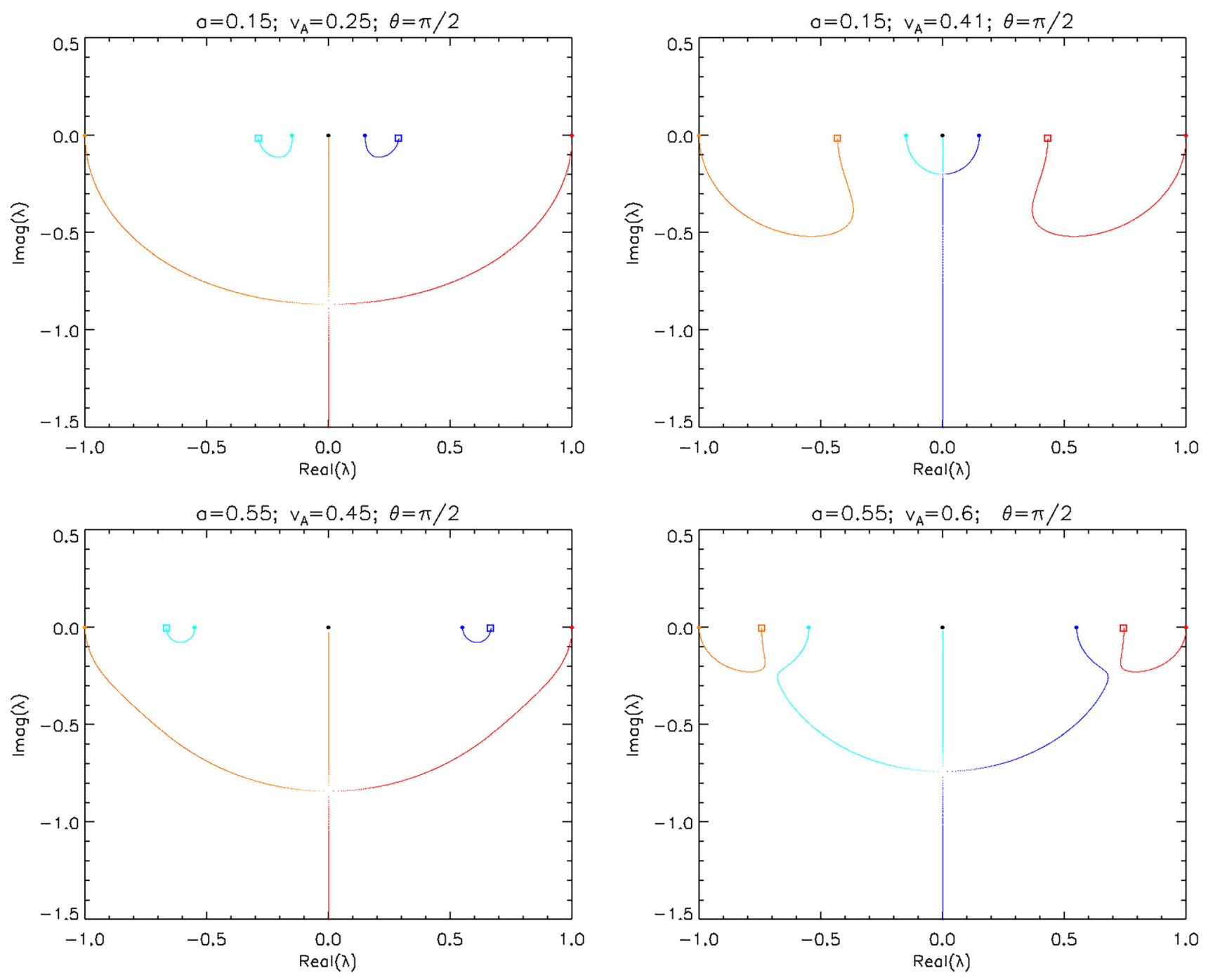

FIG. 8. Roots of $\mathcal{P}_{5}$ in the complex plane for the perpendicular case $(\theta=\pi / 2)$ : top panels correspond to the cold gas with $v_{A}=0.25,0.41$ while bottom panels refer to a hot gas and $v_{A}=0.55,0.6$ At small (large) magnetizations-left (right) panels - acoustic (light) modes are non-degenerate and tend to the magnetoacoustic solution. Plotting conventions are the same one used throughout this paper.

rightmost branch, one has a transition from type $N$ to type $P$ (e.g., slow/fast magnetosonic degeneracy). Left and right branches intersect at a cusp point which marks the appearance of a triple root (see Appendix $\mathrm{C}$ for $\theta=\pi / 2$ ).

The horizontal gray dotted line corresponds to the presence of a pair of double roots in the perpendicular case found at $u_{A}=\sqrt{a}$ [Eq. (47)]. As it will be shown shortly, this condition is nearly independent of $\theta$ and it will be used to separate the low magnetization region (where light waves may become degenerate, $u_{A} \lesssim \sqrt{a}$ ) from the high magnetization region (where acoustic waves may become degenerate, $u_{A} \gtrsim \sqrt{a}$ ).

The right panels in Fig. 9 employs color-filled contour levels to show the corresponding values of $\tilde{\sigma}$, in the $\theta-v_{A}$ plane, at which the first degeneracy point is found. Orangefilled contour levels correspond to light degeneracy points, i.e., transition from type $P$ to type $N$ modes. Likewise, bluefilled levels indicate acoustic degeneracy points. In the white region, no degeneracy is present (all roots are distinct). If a given value of $v_{A}$ and $\theta$ lies on a color-filled contour, then there exists a critical value of $\tilde{\sigma}$ for which a degeneracy occurs. This value is labeled by the corresponding contour level. A triple root exists at the boundary between a contoured and the white regions: cusp points on the left panel lie on this delimiting curve.

For $\theta=0$, degenerate roots are found only when $v_{A}$ $<1 / 3$ [in correspondence of the two values of $\tilde{\sigma}$ given by Eq. (43)]. This degeneracy affects only light modes (orange contours in the right panels), it does not depend on the sound speed and it is the same for $\mathcal{P}_{5}$ and $\mathcal{P}_{4}$. By increasing $\theta$ to $\pi / 3$, the corresponding curve encloses a larger fraction of the parameter space the extent of which now depends on the value of the sound speed. The cusp forms at larger values of $v_{A}\left(v_{A} \approx 0.6\right.$ in the hot gas case), as it is also clear from the right panels. Results change significantly at larger angles $(\theta \gtrsim 1)$ : depending on the magnetization $\left(u_{A} \lesssim \sqrt{a}\right.$ or $u_{A} \gtrsim \sqrt{a}$ ) either light or acoustic modes become degenerate for some values of $\tilde{\sigma}$ as shown by the orange and blue contours in the right panels, respectively. An overlapping region where both light and acoustic waves become type $N$ modes exists for the cold gas case (green area in the top right panel). As $\theta$ approaches $\pi / 2$ (perpendicular 

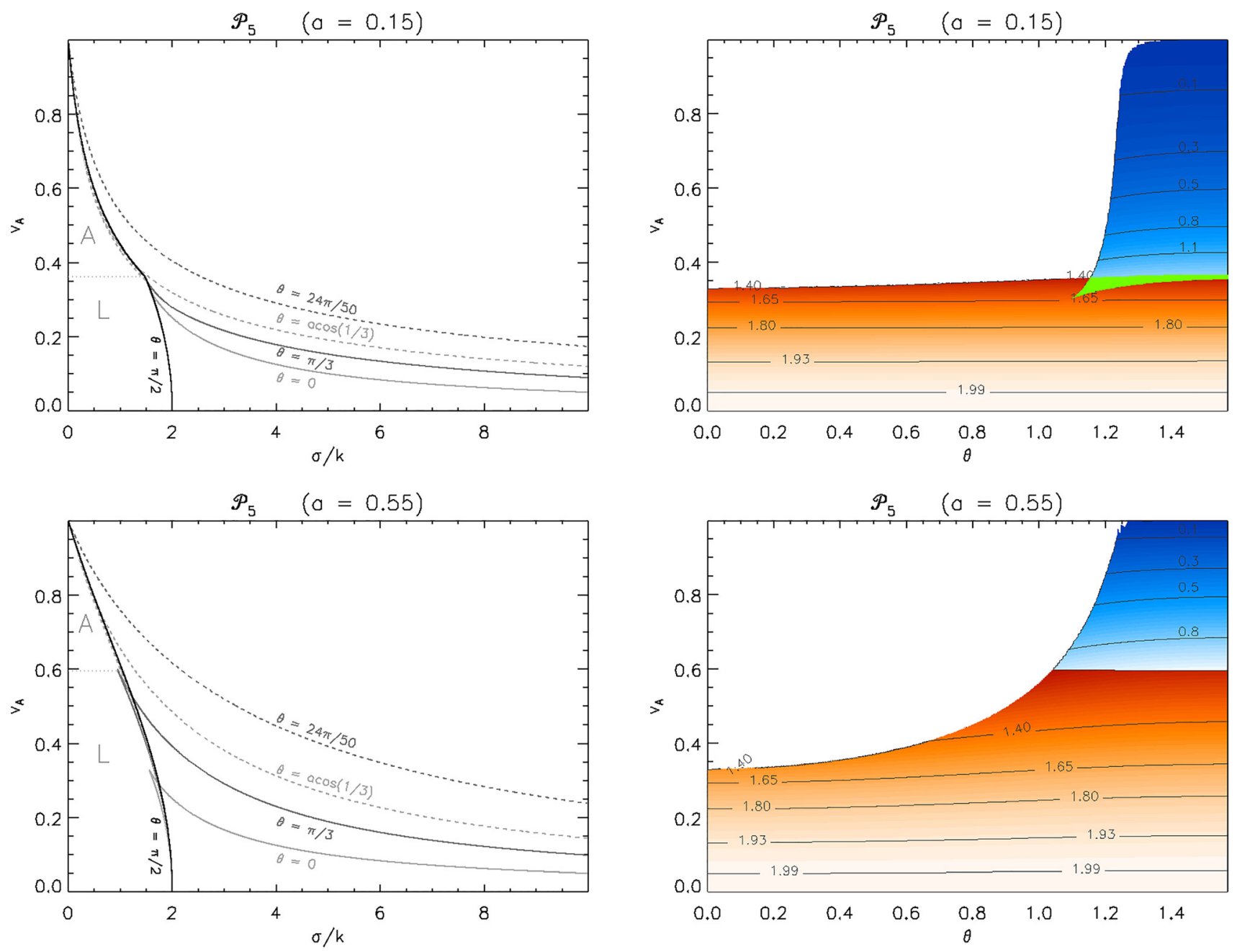

FIG. 9. Eigenmode degeneracies of $\mathcal{P}_{5}$ for arbitrary angle in the cold ( $a=0.15$, top panels) and hot $(a=0.55$, bottom panels) gas cases. The curves in the left panels show the values of $\left(\tilde{\sigma}, v_{A}\right)$ corresponding to a root of multiplicity 2 and mark a transition from a type $P$ to type $N$ mode (left branch) or vice-versa (right branch). The cusp corresponds to the formation of a triple root. Light and dark-gray solid lines correspond to $\theta=0$ and $\theta=\pi / 3$. Similar dashed lines are used for $\theta=\cos ^{-1}(1 / 3), 24 \pi / 50$. In the right panel, we show contour levels, in the $\left(\theta, v_{A}\right)$ plane, of $\tilde{\sigma}$ at which the first degeneracy (type $P$-type $\left.N\right)$ occurs. Orangefilled levels correspond to the values of $\tilde{\sigma}$ for which a light degeneracy point occurs, while blue-filled levels correspond to the acoustic degeneracy point.

propagation), a degeneracy takes place at any magnetization (dashed curves in the left panels in Fig. 9). In the limiting case $\theta=\pi / 2$, the rightmost branch of the curve becomes horizontal and stretches out to $\tilde{\sigma}=\infty$ indicating the disappearance of the slow modes.

The previous discussion can be extended to the roots of $\mathcal{P}_{4}$ using the same plotting conventions. From the left and right panels in Fig. 10, it is seen that light modes always suffer from a degeneracy (type $P$-type $N$ transition) at some critical value of $\tilde{\sigma}$ in the two following cases:

- For any $\theta \in[0, \pi / 2]$ and $v_{A}<1 / 3$ (weak magnetizations). This is a weak condition since the value of $v_{A}=1 / 3$ provides only a lower bound which we know from the case of parallel propagation [see the discussion after Eq. (43)]. The region extends indeed to larger values of $v_{A}$ as $\theta$ is increased.

- For $\theta \geq \theta_{1} \equiv \cos ^{-1}(1 / 3)$ and any value of $v_{A}$. The value $\theta_{1}$ corresponds to the intersection point between the orange-white demarcation line and the $v_{A}=1$ axis. The exact value of $\theta_{1}$ can be found by writing $\mathcal{P}_{4}$ in the limit of strong magnetization $\left(v_{A}=1\right)$,

$$
\lim _{u_{A} \rightarrow \infty} \mathcal{P}_{4}=i \tilde{\sigma} \lambda^{3}-\tilde{\sigma}^{2} \lambda^{2}-i \tilde{\sigma} \lambda+\tilde{\sigma}^{2} \cos ^{2} \theta
$$

and by imposing the condition for a perfect cubic (triple root). This yields $\cos \theta_{1}=1 / 3$ and $\tilde{\sigma}_{1}=\sqrt{3}$ and corresponds to the cusp point brushing the $v_{A}=1$ axis in the left panel of Fig. 10 (light grey solid line). Thus, for strongly magnetized plasmas $\left(v_{A} \sim 1\right)$ light modes propagating almost perpendicularly become degenerate for some value $\tilde{\sigma} \geq \sqrt{3}$.

The second degeneracy, corresponding to the type N-type $P$ transition (rightmost branch in the left panels in Fig. 10), shifts at increasingly larger values of $\tilde{\sigma}$ and it extends to infinity as $\theta \rightarrow \pi / 2$.

\section{Polar diagram}

The phase velocity of the waves can be plotted as a function of the polar angle measured from the direction of the background field $B_{0}$. Since our results are only weakly depending on the value of the sound speed, we now restrict our attention to $a=0.55$. The most prominent cases are shown in the 

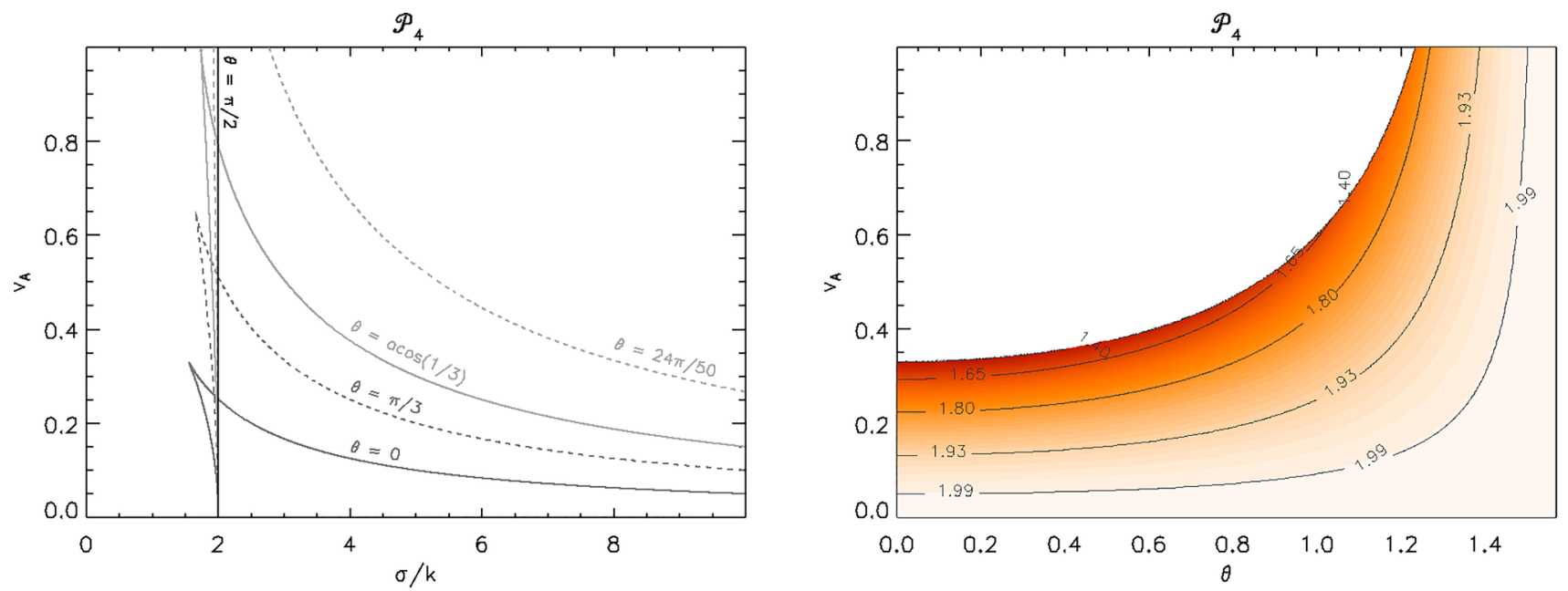

FIG. 10. Eigenmode degeneracies of $\mathcal{P}_{4}$ for arbitrary angle propagation. The same plotting convention of Fig. 9 is used.

sequence of panels Fig. 11 where polar diagrams for the roots of $\mathcal{P}_{5}$ and $\mathcal{P}_{4}$ are shown using green, red (for the former), and blue (for the latter). From left to right, we show a sequence of panels corresponding to increasing values of $\tilde{\sigma}$. From the previous discussion, a type $P$-type $N$ transition is expected around $\tilde{\sigma} \approx 2$ for a weakly magnetized plasma. For this reason, selected plots are shown using values of $\tilde{\sigma}$ immediately before and after this transition threshold.
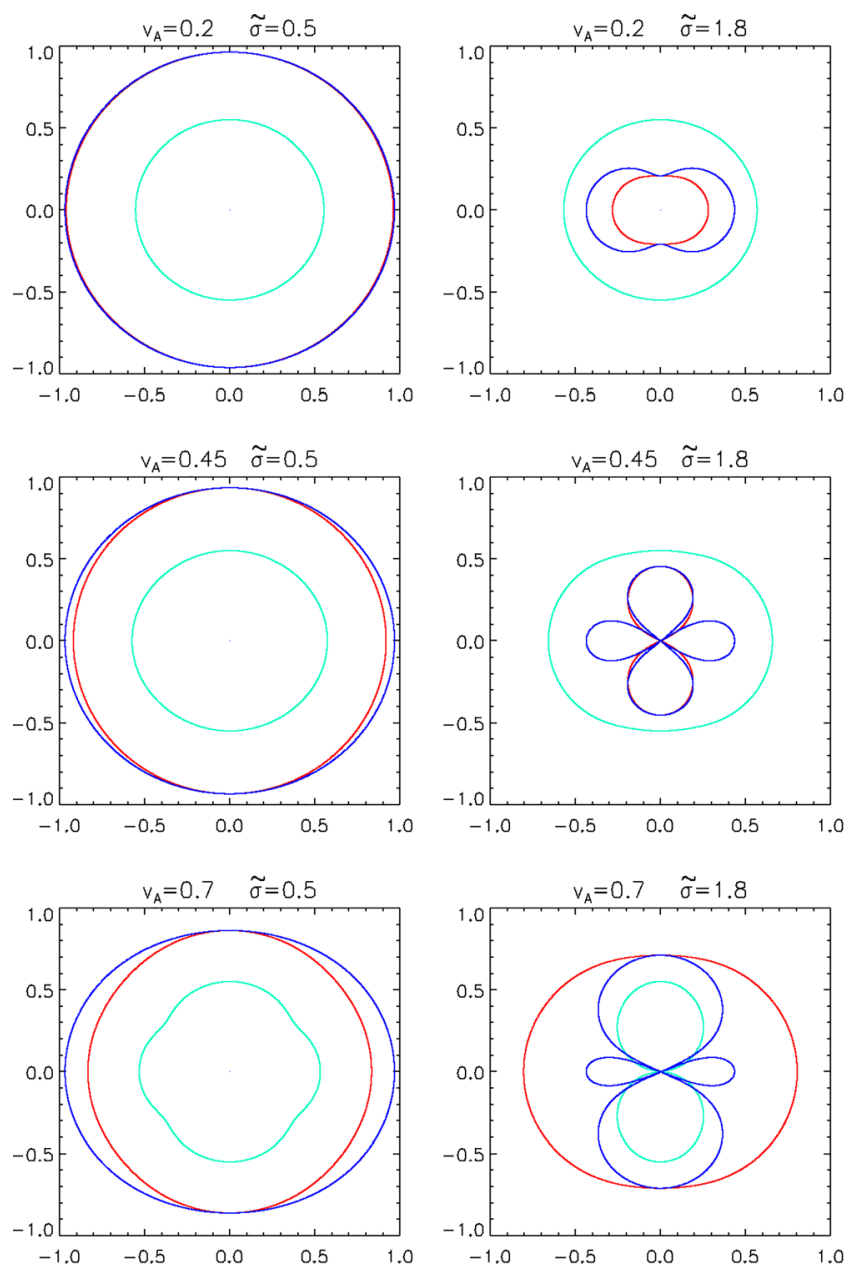

- For small values of the conductivity ( $\tilde{\sigma}=0.5$, leftmost panels in Fig. 11), signal velocities of light and acoustic modes propagate essentially isotropically with a weak dependence on the angle. The light-waves of $\mathcal{P}_{4}$ are slightly larger than those of $\mathcal{P}_{5}$ but they coincide in the case of parallel propagation $(\theta=0)$, as also shown by Eqs. (42) and (41).

- At $\tilde{\sigma}=1.8$ (second column of panels), no degeneracy is yet present for $v_{A}=0.2$ and the phase speed of the light
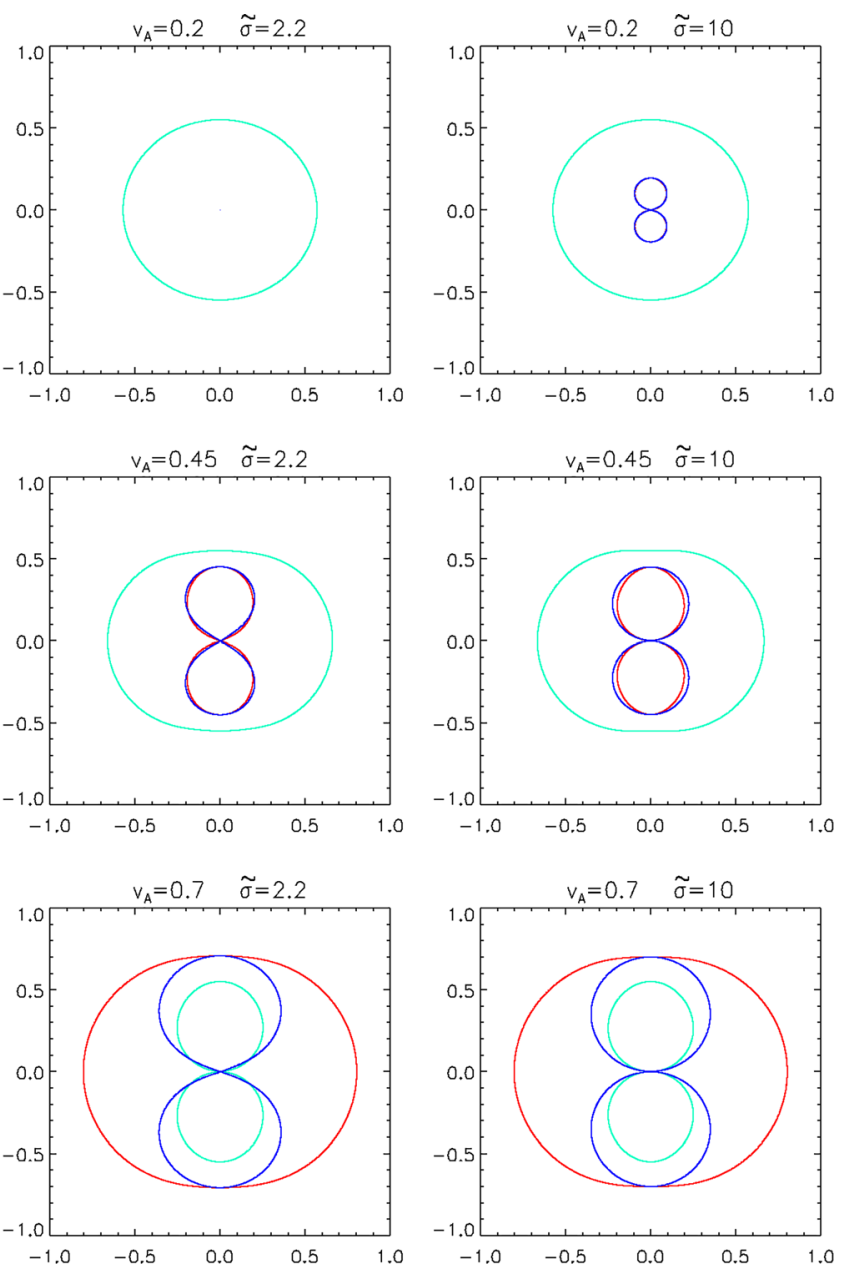

FIG. 11. Polar diagrams showing the phase velocity for different values of $\tilde{\sigma}$ (left to right) and of the magnetization parameter $v_{A}$ (top to bottom). 
modes becomes smaller than the sound speed. When the magnetization is increased at $v_{A}=0.45$, light-waves of $\mathcal{P}_{5}$ become degenerate in a narrow range around $\theta \approx \pi / 3$ (see the bottom left panel in Fig. 9), whereas acoustic waves propagate distinctly. Finally, when $v_{A}=0.7$ $>\sqrt{a /(1+a)}$, light modes are distinct and the acoustic modes are now degenerate.

In the case of $\mathcal{P}_{4}$, light modes become first degenerate at some intermediate value of $\theta(1 \lesssim \theta \lesssim 1.2$, see the right panel in Fig. 10), while roots are again distinct for larger values of $\theta$.

- For $\tilde{\sigma}=2.2$ (third column of panels), only the acoustic modes can propagate at small magnetization $\left(v_{A}=0.2\right.$, top), while all light modes have become type $N$ modes. Increasing the magnetization to $v_{A}=0.45$ (second panel from top), we see that light modes can propagate parallel to the field but become suppressed in a narrow range around $\theta \geqslant \pi / 3$. Strengthening the field to $v_{A}=0.7$ leads to the degeneracy of the acoustic modes and the $\mathcal{P}_{4}$ light modes at large angles, while light-waves of $\mathcal{P}_{5}$ are, as expected, distinct.

- For $\tilde{\sigma}=10$ (rightmost column of panels), we recover the usual ideal polar diagram for fast, slow, and Alfvén waves. Fast and slow magnetosonic modes are given by the roots of $\mathcal{P}_{5}$, while Alfvén waves are given by the roots of $\mathcal{P}_{4}$. For weak and moderate magnetizations (first and second panels from the top), the green curve identifies the fast mode (this solution is always smoothly connected to the acoustic mode), while red and blue curves are very similar and represent pairs of slow and Alfvén modes (no perpendicular propagation is allowed for these solutions). This trend reverses once the magnetization is strong enough $\left(v_{A}=0.7\right.$, third panel from the top) because of the light-acoustic degeneracy: the light modes of $\mathcal{P}_{5}$ (red) have now become fast magnetosonic waves, whereas blue and green identity, respectively, pairs of Alfvén and slow magnetosonic modes.

\section{Group, signal, and front velocity}

The explicit dependence of $\lambda$ on $\tilde{\sigma}$ obtained in Secs. IV A-IV C raises some interesting questions about the significance of the group velocity. Being the medium dissipative, the classical expression for the group velocity $v_{g}=d \omega / d k$ [see Eq. (23)] is complex, so a first question is about its physical meaning. This issue has been addressed by Muschietti and Dum $^{21}$ who showed that, because the wavenumber components are damped at different rates, the central wavenumber changes with time. The imaginary part of the group velocity accounts for this change.

A second question arises because (the real part of) $v_{g}$ may occasionally exceed unity when the real part of $\lambda$ quickly approaches zero at degenerate points or for small conductivities. An example, using $\theta=0.7, a=0.1$ and $v_{A}$ $=0.1$ is shown in Fig. 12 where we plot the group velocity for the light and acoustic waves. We remind, however, that the group velocity represents the propagation speed of an envelope which is not too broad in wave number but, in general and contrary to a diffuse misconception, it does not

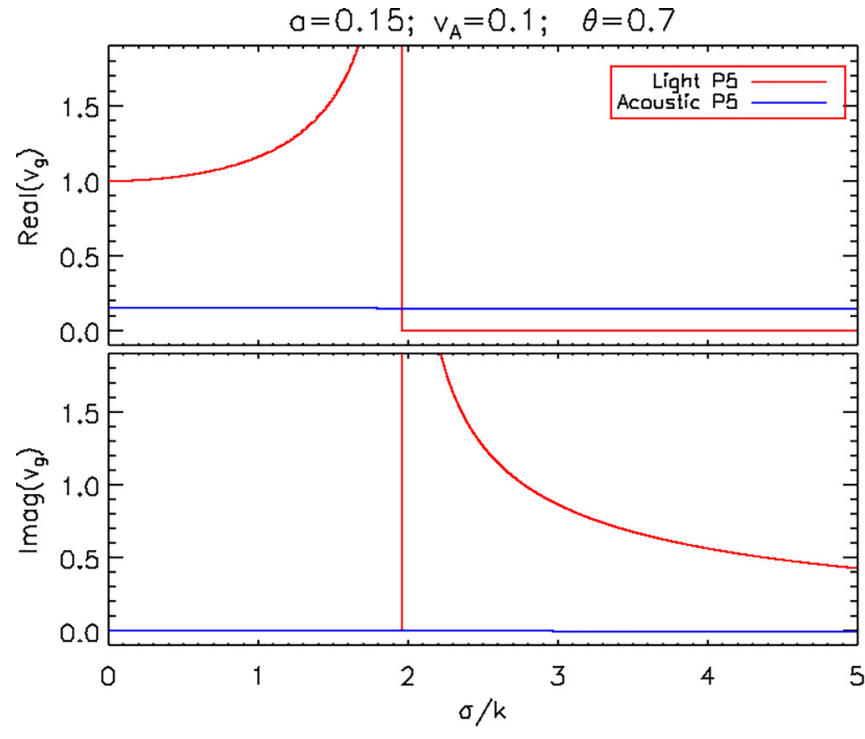

FIG. 12. Group velocities for $\mathcal{P}_{5}$ corresponding to the same parameters used in Fig. 1. Only the upper-half plane in the region $\tilde{\sigma} \in[0,5]$ is shown.

define the speed at which information travels [see, for instance, Refs. 22 (p. 337), 23, and 24 (p. 324)]. The actual signal velocity, instead, is related to the propagation of a wave packet with a finite spatial width ${ }^{25}$ or to a short isolated succession of wavelets, with the system being at rest before the signal arrives and also after it has passed. ${ }^{23}$ In this respect, a closely related concept is that of the front velocity which tracks the very first arrival of a disturbance that carries information that cannot be predicted from an earlier time. Causality cannot be violated if the front velocity is less than or equal to the speed of light.

For this purpose, we consider the special case of perpendicular propagation (Sec. IV C2) for which the dispersion relation has analytical expressions given by Eq. (50). From that expression, the group velocity is found to be

$$
v_{g}(k)= \pm \frac{2}{\sqrt{4-\tilde{\sigma}^{2}}}= \pm \frac{2 k}{\sqrt{4 k^{2}-\sigma^{2}}},
$$

which is always superluminal and even diverges for $\tilde{\sigma} \rightarrow 1 / 2$. Note also that the previous expression coincides with the expression given in Sec. IV of Ref. 14. It is easy to show that the equations for the $E_{y}$ and $B_{z}$ reduce to the telegraph equation

$$
\frac{\partial^{2} \psi}{\partial t^{2}}+\sigma \frac{\partial \psi}{\partial t}-\frac{\partial^{2} \psi}{\partial x^{2}}=0
$$

where $\psi \equiv \psi(x, t)$ stands for either $E_{y}$ or $B_{z}$. A harmonic analysis in space (see Sec. 5.10 of Ref. 22) shows that the solution of Eq. (53) is determined by the wavefunction

$$
\psi(x, t)=\frac{e^{-\sigma t / 2}}{2}\left[\psi_{0}(x+t)+\psi_{0}(x-t)+\frac{\sigma}{2} \mathcal{D}_{0}(x, t)+\mathcal{D}_{1}(x, t)\right],
$$

where $\psi_{0}(x)=\psi(x, 0)$ is the initial condition, while the $\mathcal{D}_{n}$ terms are integrals of the Bessel function of the first kind and its derivative times the initial distribution 


$$
\begin{aligned}
& \mathcal{D}_{0}(x, t)=\int_{x-t}^{x+t} \psi_{0}(\xi) J_{0}(z(x, t, \xi)) d \xi \\
& \mathcal{D}_{1}(x, t)=\int_{x-t}^{x+t} \psi_{0}(\xi) \frac{\partial}{\partial t} J_{0}(z(x, t, \xi)) d \xi
\end{aligned}
$$

with $z(x, t, \xi)=(\sigma / 2) \sqrt{(x-\xi)^{2}-t^{2}}$ (in our derivation we have set the term $\left.\partial \psi /\left.\partial t\right|_{t=0}=0\right)$. For an initial square pulse $\psi_{0}(x)=\left(1+\operatorname{sgn}\left(x_{0}-|x|\right)\right) / 2$, the wavefunction given by Eq. (54) has been computed numerically and it is plotted in Fig. 13 at different times $=0,1,2.5,4$. For this calculation, $\sigma=1 / 2$ has been used. The evolution discloses that the initial distribution splits into a pair of damped, left- and rightgoing waves. The contribution of the integrals $\mathcal{D}_{n}$ does not alter the propagation speed (the integral vanishes for $|x|$ $>\left|x_{0}\right|+t$, but it deformates the shape of the wave leaving a residue field after the front has passed through. The speeds of the two fronts always remain equal to the speed of light $(=1)$.

\section{SUMMARY}

A characteristic analysis of the resistive relativistic MHD equations has been the subject of this work. Starting from an equilibrium state describing a static and homogeneous relativistic plasma threaded by a constant magnetic field, perturbations have been introduced in the form of plane waves $\propto \exp [i(k x-\omega t)]$, where $k \in \mathbb{R}$ while $\omega \in \mathbb{C}$ is a complex quantity. The dispersion relation has been obtain as a tendegree polynomial which can be factorized into a single root $\lambda$ $=0$ and two lower-order polynomials of degree five and four, respectively. The coefficients of the two polynomials are expressed in terms of four parameters: the sound speed $a$, the magnetization $u_{A}=B_{0} / \sqrt{w_{0}}$ (or $v_{A}=u_{A} / \sqrt{1+u_{A}^{2}}$ ), the angle $\theta$ between the wavevector and the background magnetic

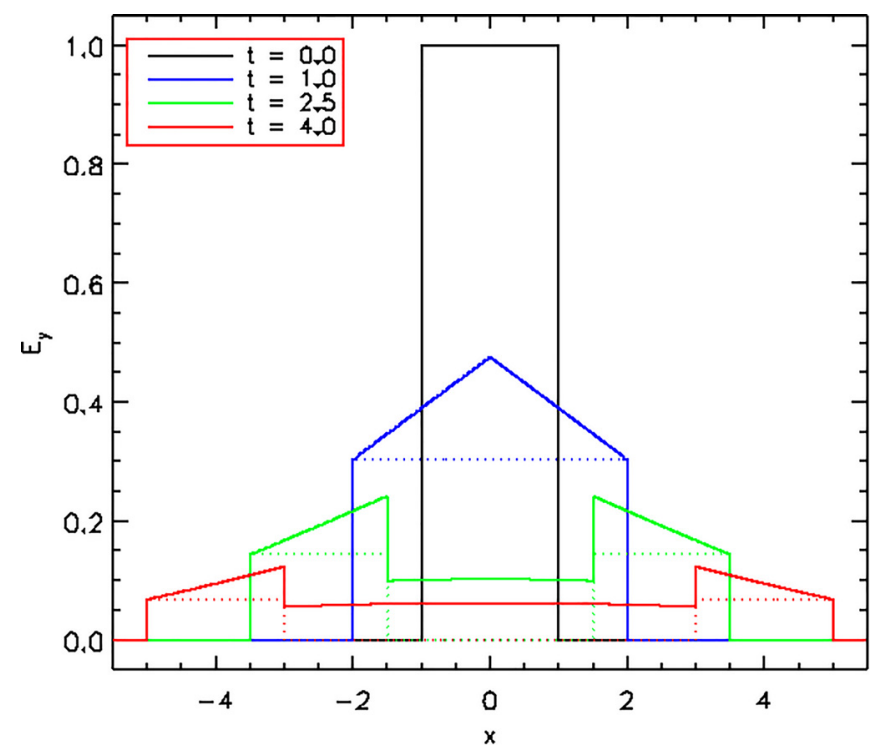

FIG. 13. Evolution of a square pulse in a dissipative dispersive media with dispersion relation given by $\omega(k)=-\frac{i}{2 \eta} \pm \sqrt{k^{2}-\frac{1}{4 \eta^{2}}}$ [see Eq. (50)] where $\eta$ $=1 / \sigma=2$. The dotted line gives the corresponding solution in an ideal medium $(\omega=k)$. field $B_{0}$, and the ratio $\sigma / k$ between the electric conductivity $\sigma$ and the wavenumber $k$.

Solution modes are of two kinds: (i) waves with nonzero phase speed which always come as pairs of opposite complex conjugate solutions or (ii) purely damped standing waves. The isolated root $\lambda=0$ coincides with the contact mode and it is unaffected by resistivity. The remaining waves can be easily identified in the fully resistive limit (zero conductivity or small wavelengths) where electromagnetic fields and matter are decoupled so that characteristic information is propagated through light or sound waves. In this limit, one has four light-waves, two acoustic waves, and three damped waves (in addition to the contact mode). In the ideal limit (infinite conductivity or large wavelengths), solution modes asymptotically approach pairs of fast, slow, or Alfvén waves (and the contact mode). Using asymptotic analysis, we have shown that the damping rates of these propagating modes scales as $\eta k^{2}$ ( $\eta$ is the plasma resistivity), as expected for a diffusive system. Conversely, the three damped modes become singular solutions of the equations and become linearly suppressed with the conductivity.

For arbitrary values of $\sigma / k$, the dispersion relation cannot be solved in a closed analytical form and a numerical approach has been employed. Our results confirm that eigenvalues are, in general, complex quantities with a negative (or zero) imaginary part indicating wave damping, a defining feature of dissipative systems. Given the nonlinear dependency on $\sigma / k$, the system is also dispersive with light waves propagating at small wavelengths while fast or slow mode propagating at large wavelengths.

In general, the solution space is characterized by a number of mode transitions which involve a root degeneracy. Isolated roots of multiplicity two define a boundary region of the parameter space inside which a pair of propagating (type $P$ ) modes has transitioned to a pair of non-propagating (type $N$ ) modes. Conversely, through a pair of double roots, solution modes switch their asymptotic behavior (e.g., light and acoustic waves interchange with each other) by remaining type $P$ modes. These transition points are described by degeneracy conditions of quintic and quartic polynomials and, in general, no simple expression has been found except for special cases. However, some general results could be established:

- For weak magnetization-namely $u_{A}<1 / \sqrt{8}$ for parallel propagation or $u_{A} \lesssim \sqrt{a}$ at larger angles - there is always a finite range of values of $\sigma / k$ where light modes degenerate into a pair of standing damped waves. On the contrary, acoustic modes remain distinct for any value of $\sigma / k$ and, in the ideal limit, they asymptotically approach the fast (when $v_{A} \lesssim a$ ) or slow magnetosonic (when $v_{A} \gtrsim a$ ) waves.

- For sufficiently stronger magnetizations and $\cos \theta \lesssim 1 / 3$, no degeneracy occurs and the four light-waves and the two acoustic modes smoothly connect to fast, slow, and Alfvén waves in the ideal limit. The magnetization threshold coincides with $u_{A}=1 / \sqrt{8}$ for parallel propagation but it increases with the inclination angle.

- As the inclination becomes more perpendicular $\left(\cos ^{-1}(1 / 3)\right.$ $\leq \theta \leq \pi / 2$ ) and $u_{A} \gtrsim \sqrt{a}$, only two light-waves remain distinct while the remaining type $P$ solutions ( 2 acoustic and 2 
light modes) always become degenerate for some intermediate value range of $\sigma / k$. In the limit of very strong magnetic fields, acoustic modes become quickly suppressed and disappear for perpendicular propagation. In the limit $\sigma / k \rightarrow \infty$, the two distinct roots smoothly connect to the fast magnetosonic modes, while the remaining ones tend to slow and Alfvén solutions.

To the extent of our knowledge, our results provide the first systematic analysis of the characteristic structure of the relativistic MHD equations in the presence of a finite conductivity. The outcome of this work may be particularly relevant in the field of relativistic magnetic reconnection as well as representing a potential benefit for the development of improved numerical methods in the solution of these kinds of equations.

\section{APPENDIX A: PURELY IMAGINARY SOLUTIONS OF THE DISPERSION RELATION}

Here, we show that $\mathcal{P}_{5}$ always admits at least one type $N$ (purely imaginary) solutions while, in the case of $\mathcal{P}_{4}$, at least two solutions of this type must be present.

\section{Proof for $\mathcal{P}_{5}$}

We seek for a solution of the type $\lambda=i Y$ in Eq. (18). Hence, it is readily found from Eq. (18) that

$$
\begin{aligned}
\mathcal{P}_{5}(i Y)= & i\left[Y^{5}+\tilde{\sigma}\left(u_{A}^{2}+1\right) Y^{4}+\left(a^{2}+1\right) Y^{3}\right. \\
& \left.\times \tilde{\sigma}\left(C^{2}+a^{2}+u_{A}^{2}\right) Y^{2}+a^{2} Y+\tilde{\sigma} C^{2}\right],
\end{aligned}
$$

where $C=a^{2} u_{A}^{2} \cos ^{2} \theta$. The polynomial inside the square brackets is a real-valued quintic function which must always possess at least one real root. Thus, $\lambda=i Y$ is a purely imaginary solution of the original polynomial.

\section{Proof for $\mathcal{P}_{4}$}

Similarly, we seek for a solution of the type $\lambda=i Y$ in Eq. (19). Upon substituting in Eq. (19) we find

$$
\begin{aligned}
\mathcal{P}_{4}(i Y)= & Y^{4}+\tilde{\sigma}\left(u_{A}^{2}+2\right) Y^{3}+\left[\left(u_{A}^{2}+1\right) \sigma^{2}+1\right] Y^{2} \\
& +\tilde{\sigma}\left(u_{A}^{2}+1\right) Y+\tilde{\sigma}^{2} u_{A}^{2} \cos ^{2} \theta .
\end{aligned}
$$

The previous equation is again a real-valued quartic equation which has the following properties:

$$
\left\{\begin{array}{l}
\lim _{Y \rightarrow-\infty} \mathcal{P}_{4}(i Y)=+\infty \\
\mathcal{P}_{4}(0)=\tilde{\sigma}^{2} u_{A}^{2} \cos ^{2} \theta \\
\mathcal{P}_{4}(-i \tilde{\sigma})=-\tilde{\sigma}^{2} u_{A}^{2} \sin ^{2} \theta .
\end{array}\right.
$$

For $\theta>0$, the quartic is positive at $Y \rightarrow-\infty$ and $Y=0$ but negative in the neighbourhood of $Y=-\tilde{\sigma}$ and thus (at least) two roots must be found in the range $Y \in[-\infty, 0]$ which proves our statement. In the special case $\theta=0$, the quartic simplifies to

$$
(Y+\tilde{\sigma})\left[Y^{3}+\tilde{\sigma}\left(u_{A}^{2}+1\right) Y^{2}+Y+\tilde{\sigma} u_{A}^{2}\right]=0,
$$

which is satisfied for $Y=-\tilde{\sigma}$ and by at least one root of the cubic inside the square brackets.

\section{APPENDIX B: EIGENVECTOR EXPRESSION IN THE RESISTIVE AND IDEAL LIMITS}

In the $\tilde{\sigma} \rightarrow 0$ (resistive) limit, Eq. (38) can still be used to obtain the eigenvectors for the compressible modes which, not surprisingly, reduce to a pair of relativistic sound waves carrying perturbations in density, pressure, and normal velocity only

$$
\left(\begin{array}{c}
\rho_{1} \\
v_{1 x} \\
p_{1}
\end{array}\right)=\left(\begin{array}{c}
1 \\
\frac{a}{\rho_{0}} \\
\frac{a^{2} w_{0}}{\rho_{0}}
\end{array}\right) .
$$

However, for the light modes, the assumption $\rho_{1}=0$ leads to a singular expression but the direct solution of Eq. (13) with $\rho_{1}=0$ provides the usual eigenvectors for Maxwell equations

$$
\left(\begin{array}{c}
B_{1 y} \\
B_{1 z} \\
E_{1 x} \\
E_{1 y} \\
E_{1 z}
\end{array}\right)=\left(\begin{array}{c}
1 \\
0 \\
0 \\
0 \\
\pm 1
\end{array}\right),\left(\begin{array}{c}
0 \\
1 \\
0 \\
\pm 1 \\
0
\end{array}\right),
$$

where $\rho_{1}=\boldsymbol{v}_{1}=p_{1}=0$.

In the ideal limit (large wavelengths or infinite conductivity), the compressible modes are given by Eq. (38) by simply taking $\sigma / k \rightarrow \infty$

$$
\left(\begin{array}{c}
\rho_{1} \\
v_{1 x} \\
v_{1 y} \\
v_{1 z} \\
B_{1 y} \\
B_{1 z} \\
E_{1 x} \\
E_{1 y} \\
E_{1 z} \\
p_{1}
\end{array}\right)=\rho_{1}\left(\begin{array}{c}
\frac{\lambda}{\rho_{0}} \\
-\frac{\lambda u_{A}^{2} \sin \theta \cos \theta\left(1-\lambda^{2}\right)}{\rho_{0} \Delta} \\
0 \\
\frac{\lambda^{2} \sqrt{w_{0}} u_{A} \sin \theta}{\rho_{0} \Delta} \\
0 \\
0 \\
0 \\
-\frac{\lambda^{3} \sqrt{w_{0}} u_{A} \sin \theta}{\rho_{0} \Delta} \\
\frac{\lambda^{2} w_{0}\left(\left(u_{A}^{2}+1\right) \lambda^{2}-u_{A}^{2}\right)}{\rho_{0} \Delta}
\end{array}\right),
$$

where now $\Delta=\lambda^{2}-\left(1-\lambda^{2}\right) u_{A}^{2} \cos ^{2} \theta$, while $\lambda$ is given by the fast and slow modes [Eq. (31)]. Incompressible perturbations are instead given by 


$$
\left(\begin{array}{c}
\rho_{1} \\
v_{1 x} \\
v_{1 y} \\
v_{1 z} \\
B_{1 y} \\
B_{1 z} \\
E_{1 x} \\
E_{1 y} \\
E_{1 z} \\
p_{1}
\end{array}\right)=B_{1 z}\left(\begin{array}{c}
0 \\
0 \\
0 \\
-\frac{\lambda \tilde{\sigma}}{B_{0} \cos \theta} \\
0 \\
1 \\
-\lambda \tan \theta \\
\lambda \\
0 \\
0
\end{array}\right),
$$

where now $\lambda$ is given by the Alfvén modes [Eq. (33)]. Moreover, Eq. (B4) reduces to the classical MHD expressions in the non-relativistic limit $w_{0} \rightarrow \rho_{0}$, where $v_{1 z}=\mp 1 /$ $\sqrt{\rho_{0}}, B_{1 z}=1$ and $\boldsymbol{E}_{1}=-\boldsymbol{v}_{1} \times \boldsymbol{B}_{0}$.

\section{APPENDIX C: TRIPLE ROOT OF $\mathcal{P}_{5}$ IN THE PERPENDICULAR CASE}

We now discuss the degenerate roots of $\mathcal{P}_{5}$ in the perpendicular case. From Eq. (44), the quartic polynomial inside square bracket can be converted to depressed form using the substitution $\lambda=i\left(Y-c_{3} / 4\right)$, where $c_{3}=\tilde{\sigma}\left(u_{A}^{2}+1\right)$. This yields

$$
f(Y)=Y^{4}+a_{2} Y^{2}+a_{1} Y+a_{0},
$$

where

$$
\begin{aligned}
a_{2}= & -\frac{3}{8}\left(u_{A}^{2}+1\right)^{2}+a^{2}+1, \\
a_{1}= & \frac{\tilde{\sigma}\left(u_{A}+1\right)}{2}\left[\frac{1}{4} \tilde{\sigma}^{2}\left(u_{A}+1\right)^{2}+\left(u_{A}-1\right)\left(1-a^{2}\right)\right], \\
a_{0}= & -\frac{3}{256}\left(u_{A}^{2}+1\right)^{4} \tilde{\sigma}^{4}+\frac{1}{16}\left(u_{A}^{2}+1\right) \\
& \times\left[1-3 a^{2}+\left(a^{2}-3\right) u_{A}^{2}\right] \tilde{\sigma}^{2}+a^{2} .
\end{aligned}
$$

Written in this form, the condition to have a triple root (see, for instance, Ref. 26) is

$$
a_{2}^{2}+12 a_{0}=0 \quad \text { and } \quad 8 a_{2}^{3}+27 a_{1}^{2}=0
$$

together with $a_{2}<0$. The first of the two conditions can be readily solved for $\tilde{\sigma}^{2}$ yielding

$$
\tilde{\sigma}^{2}=\frac{1}{3} \frac{a^{4}+14 a^{2}+1}{\left(u_{A}^{2}+1\right)\left(u_{A}^{2}+a^{2}\right)}
$$

and then inserted into the second one, giving the following biquadratic equation for $u_{A}$ :

$$
\begin{aligned}
& +8\left[a^{6}+3 a^{2}\left(a^{2}+1\right)+1\right] u_{A}^{4} \\
& +\left[a^{8}+76 a^{2}\left(a^{4}+1\right)-282 a^{4}+1\right] u_{A}^{2} \\
& +8 a^{2}\left[a^{6}+3 a^{2}\left(a^{2}+1\right)+1\right]=0 .
\end{aligned}
$$

Apart from the tedious form of the coefficients, the solution can be written

$$
u_{A}^{2}=\frac{1}{16} \frac{B \pm\left(1-a^{2}\right) C^{3 / 2}}{\left(a^{2}+1\right)^{3}} \text {, }
$$

where $\quad B=a^{8}+76 a^{2}\left(a^{4}+1\right)-282 a^{4}+1, C=\left(a^{2}+1\right)^{2}$ $-36 a^{2}$ physically admissible solutions require the argument of the square root to be positive, that is, $0 \leq a \leq 3-\sqrt{8}$. The location of the triple roots is shown by the red and orange curves in Fig. 7.

${ }^{1}$ A. Lichnerowicz, Relativistic Hydrodynamics and Magnetohydrodynamics (Benjamin, New York, 1967).

${ }^{2}$ A. M. Anile, in Relativistic Fluids and Magneto-Fluids, edited by A. M. Anile (Cambridge University Press, Cambridge, UK, 2005).

${ }^{3}$ S. S. Komissarov, MNRAS 303, 343 (1999).

${ }^{4}$ L. Del Zanna, N. Bucciantini, and P. Londrillo, Astron. Astrophys. 400, 397 (2003).

${ }^{5}$ D. Balsara, ApJS 132, 83 (2001).

${ }^{6}$ C. F. Gammie, J. C. McKinney, and G. Tóth, ApJ 589, 444 (2003).

${ }^{7}$ A. Mignone and G. Bodo, MNRAS 368, 1040 (2006).

${ }^{8}$ B. Giacomazzo and L. Rezzolla, J. Fluid Mech. 562, 223 (2006).

${ }^{9}$ S. S. Komissarov, MNRAS 382, 995 (2007); e-print arXiv:0708.0323.

${ }^{10}$ C. Palenzuela, L. Lehner, O. Reula, and L. Rezzolla, MNRAS 394, 1727 (2009); e-print arXiv:0810.1838.

${ }^{11}$ B. van der Holst, R. Keppens, and Z. Meliani, Comput. Phys. Commun. 179, 617 (2008); e-print arXiv:0807.0713.

${ }^{12}$ A. Mignone, M. Ugliano, and G. Bodo, MNRAS 393, 1141 (2009); e-print arXiv:0811.1483.

${ }^{13}$ R. Keppens and Z. Meliani, Phys. Plasmas 15, 102103 (2008); e-print arXiv:0810.2416

${ }^{14}$ S. Koide, Phys. Rev. D 78, 125026 (2008); e-print arXiv:0810.1324 [physics.plasm-ph]

${ }^{15}$ M. Takamoto and T. Inoue, ApJ 735, 113 (2011); e-print arXiv:1105.5683 [astro-ph.HE].

${ }^{16}$ L. Del Zanna, E. Papini, S. Landi, M. Bugli, and N. Bucciantini, MNRAS 460, 3753 (2016); e-print arXiv:1605.06331 [astro-ph.HE].

${ }^{17}$ T.-P. Liu, Commun. Math. Phys. 108, 153 (1987).

${ }^{18} \mathrm{~J}$. A. Hittinger, "Foundations for the generalization of the Godunov method to hyperbolic systems with stiff relaxation source terms," $\mathrm{Ph} . \mathrm{D}$. thesis (University of Michigan, 2000).

${ }^{19}$ P. L. Roe and J. A. F. Hittinger, "Toward Godunov-type methods for hyperbolic conservation laws with stiff relaxation," in Godunov Methods. Theory and Applications, edited by E. F. Toro (Springer US, Boston, MA, 2001), pp. 725-744.

${ }^{20}$ E. Durand, Solutions Numériques des Équations Algébriques (Masson, Paris, 1971).

${ }^{21}$ L. Muschietti and C. T. Dum, Phys. Fluids B 5, 1383 (1993).

${ }^{22}$ J. A. Stratton, Electromagnetic Theory, International Series in Pure and Applied Physics (McGraw-Hill, New York, NY, 1941).

${ }^{23}$ Wave Propagation and Group Velocity, Pure and Applied Physics, Vol. 8, edited by L. Brillouin (Elsevier, 1960), pp. 1-16.

${ }^{24}$ J. D. Jackson, Classical Electrodynamics, 3rd ed., edited by J. D. Jackson (Wiley-VCH, 1998), p. 832, ISBN 0-471-30932-X.

${ }^{25}$ A. Sommerfeld, in Wave Propagation and Group Velocity, Pure and Applied Physics, Vol. 8, edited by L. Brillouin (Elsevier, 1960), pp. 17-42.

${ }^{26} \mathrm{R}$. Viher, KoG 11, 25 (2007). 